\title{
Role of a four-quark and a glueball state in pion-pion and pion-nucleon scattering
}

\author{
Phillip Lakaschus, ${ }^{1}$ Justin L. P. Mauldin, ${ }^{1}$ Francesco Giacosa, ${ }^{2}$ and Dirk H. Rischke ${ }^{1,3}$ \\ ${ }^{1}$ Institute for Theoretical Physics, Goethe University, Max-von-Laue-Straße 1, D-60438 Frankfurt am Main, Germany \\ ${ }^{2}$ Institute of Physics, Jan Kochanowski University, PL-25406 Kielce, Poland \\ ${ }^{3}$ Interdisciplinary Center for Theoretical Study and Department of Modern Physics, University of Science and Technology of China, \\ Hefei, Anhui 230026, China
}

(Received 28 July 2018; published 8 April 2019)

\begin{abstract}
We consider the two-flavor version of the extended linear sigma model (eLSM), which contains (pseudo)scalar and (axial-)vector quark-antiquark mesons, a scalar glueball [predominantly corresponding to $f_{0}(1710)$ ], as well as the nucleon and its chiral partner. We extend this model by the additional light scalar meson $f_{0}(500)$, predominantly a putative four-quark state. We investigate various interaction terms of the four-quark and glueball states with the other particles, some of which preserve and some of which explicitly break the $U(1)_{A}$ symmetry. We test our model by performing a global fit to masses and decay widths of the scalar resonances and pion-pion scattering lengths. We also discuss the influence of the scalar four-quark state and the glueball on the baryon sector by evaluating pion-nucleon scattering parameters. We find that the inclusion of $f_{0}(500)$ improves the description of pion-pion and pion-nucleon scattering lengths.
\end{abstract}

DOI: 10.1103/PhysRevC.99.045203

\section{INTRODUCTION}

A major task in low-energy hadron physics is the unified description of masses, decays, and scattering properties (including scattering lengths, phase shifts, etc.) of all light hadrons (both mesons and baryons) below $\approx 2 \mathrm{GeV}$ [1]. This problem is exceptionally difficult, due to the large number of hadrons and the intrinsically strong interaction between them.

Since quantum chormodynamics (QCD), the fundamental theory of the strong interaction, cannot be directly solved in the low-energy domain, various methods were developed to describe mesons and baryons. The relativistic quark model of Refs. [2,3] solidly reproduces properties of conventional quark-antiquark and three-quark states. Even after many years, it still provides a useful starting point for many considerations. Yet, the effect of mesonic quantum corrections is not taken into account (for an extension in this direction, see Ref. [4]) and various candidates for nonconventional mesons (such as glueballs, hybrids, and multiquark states) cannot be easily accounted for. On the other hand, numerical simulations of QCD on the lattice are now capable of reproducing a large part of the QCD spectrum (see, e.g., Ref. [5]). Nowadays even scattering lengths can be computed; see, e.g., Ref. [6]. However, there is still a long way to go towards an exhaustive description of all properties of low-energy QCD using lattice simulations.

Published by the American Physical Society under the terms of the Creative Commons Attribution 4.0 International license. Further distribution of this work must maintain attribution to the author $(s)$ and the published article's title, journal citation, and DOI. Funded by $S C O A P^{3}$.
Another line of research has been the development of effective chiral approaches. Some make use of quark degrees of freedom, such as the famous Nambu-Jona-Lasinio (NJL) model [7-11] (and the related quark-meson model; see Refs. [12-14] and references therein). More recently, Dyson-Schwinger equations have been employed to calculate meson and baryon masses in an approach which starts directly from the QCD Lagrangian and respects chiral symmetry (for reviews, see Refs. $[15,16])$.

Another approach to describe meson properties in the low-energy domain is chiral perturbation theory (ChPT); see, e.g., Refs. [17-21]. It is based on a nonlinear realization of chiral symmetry and the primary method to study hadronic low-energy properties in a systematic and well-defined way. ChPT is originally devised to study the interactions of the (pseudo-)Goldstone bosons emerging from chiral symmetry breaking, i.e., for two quark flavors the pions. The description of other (and heavier) mesons becomes more difficult [22,23].

Other chiral approaches, so-called linear sigma models (see, e.g., Refs. [24-29]), are based on the linear realization of chiral symmetry, hence they contain hadrons and their chiral partners on the same footing. In particular, within the last ten years a chiral model, called the extended linear sigma model (eLSM), has been developed $[27,28,30]$ in an attempt to include as many resonances as possible. The eLSM is based on both chiral symmetry and dilatation invariance and correctly models their respective explicit, anomalous, and spontaneous breaking mechanisms. In the mesonic sector, the eLSM contains also (axial-)vector meson degrees of freedom besides the standard (pseudo)scalar mesons. The lightest scalar glueball is included as a dilaton field in the Lagrangian. Moreover, the model was extended to include the lightest pseudoscalar glueball [31], a vector glueball, pseudovector and excited vector mesons [32], as well as excited (pseudo)scalar mesons [33]. 
In the low-energy limit, the eLSM correctly reduces to ChPT [34], thus showing the compatibility of these two different approaches to hadronic physics. In the baryonic sector, the eLSM was developed for two flavors in Ref. [35] and for three flavors in Ref. [36] on the basis of the mirror assignment for the chiral partner of the nucleon [37], in such a way that chirally invariant mass terms for baryons are possible.

In general, the eLSM offers a satisfactory description of hadronic properties below $2 \mathrm{GeV}$ (see Ref. [28]). In particular, the eLSM has a clear answer concerning the interpretation of scalar mesons [30]: the chiral partner of the pion is predominantly $f_{0}(1370)$, its quark structure being $(\bar{u} u+\bar{d} d) / \sqrt{2}$, while $f_{0}(1500)$ is predominantly $\bar{s} s$. Then, the state $f_{0}(1710)$ is mostly gluonic and could be the lightest scalar glueball in agreement with calculations within lattice QCD [38,39] and holographic QCD [40-43] (for other interpretations suggesting a mixing of quarkonium states, see Refs. [44-47] and references therein). The isovector state $a_{0}(1450)$ and the isodoublet states $K_{0}^{*}(1430)$ complete the nonet of $\bar{q} q$ states, respectively. In this way, the light scalar resonances $f_{0}(500), f_{0}(980), a_{0}(980)$, and $K_{0}^{*}(800)$ are not part of the eLSM. Hence, these resonances are not predominantly quarkantiquark states, but something else.

There is nowadays consensus that these resonances are most likely four-quark states. This still leaves different possibilities for the internal structure of these states: following the original proposal by Jaffe [48], they could be bound states formed by a colored diquark (in the antisymmetric colorantitriplet and antisymmetric flavor-antitriplet representation) and a colored antidiquark (in the corresponding color-triplet and flavor-triplet representation) [49-52]. In this picture, the resonance $f_{0}(500)$ is a $[u, d][\bar{u}, \bar{d}]$ four-quark state. The other members of the nonet are formed similarly using also [us], $[d s]$, and the corresponding antidiquarks.

Alternatively, the light scalar mesons could be (loosely bound) molecular states formed from, or unbound states in the scattering continuum of [53-62], two color-neutral mesons. The latter possibility is supported by studies where they emerge as companion poles of conventional $\bar{q} q$ seed states [4,63-67] (for the dynamical generation of $a_{0}(980)$ and $K_{0}^{*}(800)$ starting from an eLSM-inspired Lagrangian, see Refs. [68,69]). Thus, even if the above mentioned approaches differ in the interpretation of the internal structure, all agree on a predominantly four-quark nature of the light scalars. In this respect, the findings of the eLSM are consistent with these results.

The scalar state $f_{0}(500)$ is particularly important since it is the lightest state with the quantum numbers of the vacuum (for a review, see Ref. [62]). It is expected to be relevant both in pion-pion, pion-nucleon, as well as nucleon-nucleon scattering. However, this state was not yet included in the eLSM, although some preliminary attempts were made in studies at nonzero density [70], at nonzero temperature [71], and of neutron-proton scattering [72]. Moreover, in a comparison of the eLSM with ChPT it was recently stressed that the $f_{0}(500)$ is necessary for a proper description of the pion-pion scattering lengths [34].

The main goal of the present work is the inclusion and systematic investigation of the light four-quark state $f_{0}(500)$ within the eLSM. To this end, we consider masses, decay widths, as well pion-pion and pion-nucleon scattering lengths, where $f_{0}(500)$ plays a decisive role. At the same time, we shall also investigate the effects of the glueball/dilaton field [identified with $f_{0}(1710)$ ] on these quantities. We show that the presence of both $f_{0}(500)$ and $f_{0}(1710)$ offers a satisfactory description of experimental results in the meson sector and, in the baryon sector, at least an improved description of data in comparison to models without these states.

This paper is organized as follows: in Sec. II we couple a four-quark field [which is the predominant component of $f_{0}(500)$ ] both to the mesonic and to the baryonic sector of the eLSM as an additional chirally invariant scalar (thus, in order to avoid double counting one should not generate it via loop contributions within this approach). For the sake of definiteness, we will use the diquark-antidiquark picture of this state in our considerations, but this is actually of secondary importance for the two-flavor version of the eLSM studied here.

In the baryonic sector, we make use of the so-called mirror assignment, first proposed in Ref. [37] and further studied in Refs. [35,73-79]; here, the condensation of $f_{0}(500)$ (corresponding to a four-quark condensate) and of the dilaton $f_{0}(1710)$ (corresponding to a gluon condensate) contribute to the baryonic mass terms of the mirror model in addition to the condensation of the standard quark-antiquark meson $f_{0}(1370)$.

Then, in Sec. III we present our results for masses, decay widths, and pion-pion as well as pion-nucleon scattering lengths and volumina. Here, one observes that $f_{0}(500)$ is crucial to obtain a correct description of data for the pion-pion scattering length $a_{0}^{0}$ and at least an improved description of data for the pion-nucleon scattering length $a_{0}^{(+)}$. Moreover, a detailed study of mixing between a bare four-quark state, a bare quark-antiquark state, and a bare glueball confirms that the field $f_{0}(500)$ is mostly a four-quark state, $f_{0}(1370)$ is mostly quarkonium, and $f_{0}(1710)$ is mostly gluonic. We conclude this work with a summary and a discussion of the results in Sec. IV. Details of the calculations are deferred to the Appendix. We use natural units $\hbar=c=1$; the convention for the metric tensor of flat Minkowski space-time is $g_{\mu \nu}=$ $\operatorname{diag}(+,-,-,-)$.

\section{THE MODEL}

\section{A. Quarkonium multiplets}

For an arbitrary number of flavors we can arrange (pseudo)scalar quarkonium fields into multiplets using the current

$$
\Phi^{i j} \equiv \bar{q}_{\mathrm{R}}^{i} q_{\mathrm{L}}^{j}
$$

which is a matrix in flavor space and where the color indices are implicitly contracted. This is a so-called heterochiral [80] scalar because it transforms under the global chiral symmetry $G_{\mathrm{fl}} \times \mathrm{U}(1)_{\mathrm{A}}=\mathrm{SU}\left(N_{\mathrm{f}}\right)_{\mathrm{L}} \times \mathrm{SU}\left(N_{\mathrm{f}}\right)_{\mathrm{R}} \times \mathrm{U}(1)_{\mathrm{A}}$ as

$$
\Phi \longrightarrow e^{-2 i \alpha} U_{\mathrm{L}} \Phi U_{\mathrm{R}}^{\dagger},
$$


where $U_{\mathrm{L}, \mathrm{R}}$ are $\mathrm{SU}\left(N_{\mathrm{f}}\right)_{\mathrm{L}, \mathrm{R}}$ transformations and $\alpha$ is the parameter of the U(1) A transformation. For two flavors $\Phi$ can be written as

$$
\Phi=\sum_{a=0}^{3} \Phi^{a} t^{a}=(\sigma+i \eta) t^{0}+\left(\vec{a}_{0}+i \vec{\pi}\right) \cdot \vec{t},
$$

where $t^{a}=\tau^{a} / 2$ are the generators of $\mathrm{U}(2)$, namely half the Pauli matrices for $a=1,2,3$, and half the unit matrix for $a=0$. Similarly, we define the right- and left-handed vector currents,

$$
R_{\mu}^{i j} \equiv \bar{q}_{\mathrm{R}}^{i} \gamma_{\mu} q_{\mathrm{R}}^{j}, \quad L_{\mu}^{i j} \equiv \bar{q}_{\mathrm{L}}^{i} \gamma_{\mu} q_{\mathrm{L}}^{j} .
$$

These are homochiral multiplets, i.e., they transform under the chiral symmetry $G_{\mathrm{fl}} \times \mathrm{U}(1)_{\mathrm{A}}$ as

$$
L_{\mu} \longrightarrow U_{\mathrm{L}} L_{\mu} U_{\mathrm{L}}^{\dagger}, \quad R_{\mu} \longrightarrow U_{\mathrm{R}} R_{\mu} U_{\mathrm{R}}^{\dagger}
$$

For two flavors the (axial-)vector fields are contained in the right- and left-handed meson matrices

$$
\begin{aligned}
& R_{\mu}=\sum_{a=0}^{3} R_{\mu}^{a} t^{a}=\left(\omega_{\mu}-f_{1, \mu}\right) t^{0}+\left(\vec{\rho}_{\mu}-\vec{a}_{1, \mu}\right) \cdot \vec{t}, \\
& L_{\mu}=\sum_{a=0}^{3} L_{\mu}^{a} t^{a}=\left(\omega_{\mu}+f_{1, \mu}\right) t^{0}+\left(\vec{\rho}_{\mu}+\vec{a}_{1, \mu}\right) \cdot \vec{t} .
\end{aligned}
$$

These non-exotic multiplets lead to the well-known chirally invariant Lagrangian $[28,34,35]$

$$
\begin{aligned}
\mathcal{L}_{\mathrm{eLSM}}= & \operatorname{Tr}\left[\left(D_{\mu} \Phi\right)^{\dagger}\left(D^{\mu} \Phi\right)-\mu^{2} \frac{G^{2}}{G_{0}^{2}} \Phi^{\dagger} \Phi-\lambda_{2}\left(\Phi^{\dagger} \Phi\right)^{2}\right]-\lambda_{1}\left(\operatorname{Tr}\left[\Phi^{\dagger} \Phi\right]\right)^{2}+c\left(\operatorname{det} \Phi^{\dagger}+\text { H.c. }\right)+h_{0} \operatorname{Tr}\left[\Phi^{\dagger}+\Phi\right] \\
& -\frac{1}{4} \operatorname{Tr}\left[\left(L^{\mu \nu}\right)^{2}+\left(R^{\mu \nu}\right)^{2}\right]+\frac{m_{1}^{2}}{2} \frac{G^{2}}{G_{0}^{2}} \operatorname{Tr}\left[\left(L^{\mu}\right)^{2}+\left(R^{\mu}\right)^{2}\right]+\frac{h_{1}}{2} \operatorname{Tr}\left[\Phi^{\dagger} \Phi\right] \operatorname{Tr}\left[\left(L^{\mu}\right)^{2}+\left(R^{\mu}\right)^{2}\right]+h_{2} \operatorname{Tr}\left[\Phi^{\dagger} L_{\mu} L^{\mu} \Phi\right. \\
& \left.+\Phi R_{\mu} R^{\mu} \Phi^{\dagger}\right]+2 h_{3} \operatorname{Tr}\left[\Phi R_{\mu} \Phi^{\dagger} L^{\mu}\right]+i \frac{g_{2}}{2}\left(\operatorname{Tr}\left[L_{\mu \nu}\left[L^{\mu}, L^{\nu}\right]\right]+\operatorname{Tr}\left[R_{\mu \nu}\left[R^{\mu}, R^{\nu}\right]\right]\right)+\mathcal{L}_{g_{3}, g_{4}, g_{5}, g_{6}}-V_{\mathrm{dil}}(G),
\end{aligned}
$$

where $D_{\mu}=\partial_{\mu}+i g_{1}\left(\Phi R_{\mu}-L_{\mu} \Phi\right)$ and

$$
V_{\text {dil }}(G)=\frac{1}{4} \frac{m_{G}^{2}}{\Lambda_{\text {dil }}^{2}} G^{4}\left(\ln \left|\frac{G}{\Lambda_{\text {dil }}}\right|-\frac{1}{4}\right)
$$

is the dilaton potential, responsible for the breaking of dilatation symmetry (trace anomaly) [81-84]. The scalar glueball with mass $m_{G}$ emerges upon the shift $G \rightarrow G_{0}+G$. The scalar glueball represents a color-neutral gluonic bound state. Since it is not composed of any quarks of any flavor, it is trivially a chiral singlet.

The Lagrangian $\mathcal{L}_{g_{3}, g_{4}, g_{5}, g_{6}}$ describes (axial-)vector meson interactions; see the Appendix of Ref. [35]. After chiral symmetry breaking and a shift of the axial-vector fields to eliminate bilinear mixing terms between the latter and the pions, derivatively coupled four-pion interactions emerge. Therefore, in principle the coupling constants $g_{3}, g_{4}, g_{5}, g_{6}$ have an influence on the pion-pion scattering lengths. However, it was shown in Ref. [34] that varying the values for these coupling constants between \pm 100 (i.e., a range of values that is in agreement with ChPT and moreover appears to be of a natural order of magnitude) the change in the pion-pion scattering lengths is only of the order of a few percent. Therefore, we will neglect these coupling terms in the following.

\section{B. Four-quark multiplets}

There are several ways to incorporate four-quark states into a chiral model. Here, we will follow the approach of Ref. [85]. We start with the three-flavor case and then reduce it to two flavors. There are several reasons for choosing this approach:
(1) Writing down all relevant terms for three flavors facilitates an extension of the current work to the case of three flavors.

(2) We will be able to compare our approach with other ones, e.g., those of Refs. [86,87].

(3) It is easier to see which terms are large- $N_{c}$ dominant, enabling us to choose only the most relevant terms.

(4) For three flavors a four-quark nonet has the same chiral structure as the quarkonium nonet, while for $N_{\mathrm{f}} \neq 3$ the four-quark multiplet will have a different chiral structure, which makes three-flavor models somewhat special when considering four-quark states [88].

Here, we use a diquark-antidiquark picture as a concrete framework to construct the multiplet of light scalars and to couple them to conventional mesons. However, it is also possible to construct the same terms in the meson-meson molecular picture [87]. In the two-flavor eLSM both approaches yield the same effective Lagrangian at leading order in the large- $N_{c}$ expansion. In general, when we refer to four-quark states we understand both diquark-antidiquark as well as meson-meson components; even if the latter are expected to be dominant [62], an admixture of the former configurations is possible.

For $N_{\mathrm{f}}=3$ the right- and left-handed diquark fields are defined as

$$
\begin{aligned}
& L_{c C}=\epsilon_{c a b} \epsilon_{C A B} q_{a A}^{\mathrm{T}} C \mathcal{P}_{\mathrm{L}} q_{b B}, \\
& R_{c C}=\epsilon_{c a b} \epsilon_{C A B} q_{a A}^{\mathrm{T}} C \mathcal{P}_{\mathrm{R}} q_{b B},
\end{aligned}
$$

with the charge-conjugation matrix $C=i \gamma^{2} \gamma^{0}$ (in the Dirac representation), where the flavor indices are in small letters and color indices in capital letters. For the sake of simplicity 
we will drop the color indices in the following. In the next step, we construct the right- and left-handed diquark matrices

$$
D_{\mathrm{L}, a b}=\left(A_{c}\right)_{a b} L_{c}, \quad D_{\mathrm{R}, a b}=\left(A_{c}\right)_{a b} R_{c},
$$

where $\left(A_{c}\right)_{a b} \equiv \epsilon_{c a b}$. They transform as $e^{-2 i \alpha} U_{\mathrm{L}} D_{\mathrm{L}} U_{\mathrm{L}}^{\mathrm{T}}$ and $e^{2 i \alpha} U_{\mathrm{R}} D_{\mathrm{R}} U_{\mathrm{R}}^{\mathrm{T}}$ under $G_{\mathrm{fl}} \times \mathrm{U}(1)_{\mathrm{A}}$, respectively. Under parity $D_{\mathrm{L} / \mathrm{R}}$ transforms into $-D_{\mathrm{R} / \mathrm{L}}$ and under charge conjugation into $-D_{\mathrm{R} / \mathrm{L}}^{\dagger}$, such that we obtain diquark matrices with well defined parity via the linear combinations

$$
D=\frac{D_{\mathrm{R}}-D_{\mathrm{L}}}{\sqrt{2}}, \quad \tilde{D}=\frac{D_{\mathrm{R}}+D_{\mathrm{L}}}{\sqrt{2}} .
$$

These are composed of scalar and pseudoscalar diquarks, defined as

$$
\begin{aligned}
& S_{c C}=\frac{1}{\sqrt{2}} \epsilon_{c a b} \epsilon_{C A B} q_{a A}^{T} C \gamma^{5} q_{b B}, \\
& P_{c C}=\frac{1}{\sqrt{2}} \epsilon_{c a b} \epsilon_{C A B} q_{a A}^{T} C q_{b B},
\end{aligned}
$$

such that (again suppressing color indices)

$$
S_{c}=\frac{R_{c}-L_{c}}{\sqrt{2}}, \quad P_{c}=\frac{R_{c}+L_{c}}{\sqrt{2}} .
$$

In the following we will only be interested in scalar diquarks, assuming that the pseudoscalar ones are not relevant for lowenergy hadron phenomenology. A strong attraction between two quarks in a color antitriplet $\left(\overline{3}_{\mathrm{C}}\right)$, a flavor antitriplet $\left(\overline{3}_{\mathrm{F}}\right)$, and a spin-zero configuration [48] is obtained in studies based on one-gluon exchange [89], instantons [90,91], the NJL model [92], and Dyson-Schwinger equation (DSE) [93]. In these studies it is also shown that the pseudoscalar diquark turns out to be considerably heavier.

Considering only scalar diquarks, we can therefore construct a scalar four-quark nonet by

$$
T_{a b}=S_{a}^{\dagger} S_{b},
$$

which explicitly reads

$$
\begin{aligned}
T & =\left(\begin{array}{ccc}
{[\bar{d}, \bar{s}][d, s]} & {[\bar{d}, \bar{s}][u, s]} & {[\bar{d}, \bar{s}][u, d]} \\
{[\bar{u}, \bar{s}][d, s]} & {[\bar{u}, \bar{s}][u, s]} & {[\bar{u}, \bar{s}][u, d]} \\
{[\bar{u}, \bar{d}][d, s]} & {[\bar{u}, \bar{d}][u, s]} & {[\bar{u}, \bar{d}][u, d]}
\end{array}\right) \\
= & \left(\begin{array}{ccc}
\sqrt{\frac{1}{2}}\left(\chi_{\mathrm{s}}-a_{0}^{0}\right) & -a_{0}^{+} & K_{0}^{*+} \\
-a_{0}^{-} & \sqrt{\frac{1}{2}}\left(\chi_{\mathrm{s}}+a_{0}^{0}\right) & -K_{0}^{* 0} \\
K_{0}^{*-} & -\bar{K}_{0}^{* 0} & \chi
\end{array}\right),
\end{aligned}
$$

where $a_{0}$ and $K_{0}^{*}$ are identified with $a_{0}(980)$ and $K_{0}^{*}(800)$, and admixtures of $\chi$ and $\chi_{\mathrm{s}}$ are assigned to the physical states $f_{0}(500)$ and $f_{0}(980)$. Now we are able to construct chirally invariant interaction terms that couple scalar four-quark states to scalar quarkonia:

$$
\begin{aligned}
\mathcal{L}_{\mathrm{T} \Phi \Phi}= & g_{\chi}^{(1)} \frac{G}{G_{0}} \operatorname{Tr}\left[D_{\mathrm{R}} \Phi^{\mathrm{T}} D_{\mathrm{L}}^{\dagger} \Phi+D_{\mathrm{L}} \Phi^{*} D_{\mathrm{R}}^{\dagger} \Phi^{\dagger}\right] \\
& +g_{\chi}^{(2)} \frac{G}{G_{0}} \operatorname{Tr}\left[D_{\mathrm{R}} D_{\mathrm{R}}^{\dagger} \Phi^{\dagger} \Phi+D_{\mathrm{L}} D_{\mathrm{L}}^{\dagger} \Phi \Phi^{\dagger}\right] \\
& +g_{\chi}^{(3)} \frac{G}{G_{0}} \operatorname{Tr}\left[D_{\mathrm{R}} D_{\mathrm{R}}^{\dagger}+D_{\mathrm{L}} D_{\mathrm{L}}^{\dagger}\right] \operatorname{Tr}\left[\Phi \Phi^{\dagger}\right]
\end{aligned}
$$

In each term there is a diquark and an antidiquark such that the expression becomes color neutral. Furthermore, all terms are also invariant under parity, charge conjugation, and $\mathrm{U}(1)_{\mathrm{A}}$ transformations. In order to keep our effective model as simple as possible, in the following we will only consider the first term in Eq. (19), which is the leading one in the large- $N_{\mathrm{c}}$ expansion:

$$
\begin{aligned}
\mathcal{L}_{\mathrm{T} \Phi \Phi} & =-\frac{g_{\chi}^{(1)}}{2} \frac{G}{G_{0}} \operatorname{Tr}\left[D \Phi^{\mathrm{T}} D^{\dagger} \Phi+D \Phi^{*} D^{\dagger} \Phi^{\dagger}\right]+\cdots \\
& =-\frac{g_{\chi}^{(1)}}{2} \frac{G}{G_{0}} T_{a b} \operatorname{Tr}\left[A_{b} \Phi^{\mathrm{T}} A_{a}^{\mathrm{T}} \Phi+A_{b} \Phi^{*} A_{a}^{\mathrm{T}} \Phi^{\dagger}\right]+\cdots,
\end{aligned}
$$

where we used Eqs. (13) and (17) and neglected all terms containing pseudoscalar diquarks. ${ }^{1}$

For two flavors, Eq. (20) reduces to

$$
\begin{aligned}
\mathcal{L}_{\mathrm{T} \Phi \Phi} & =2 g_{\chi} \frac{G}{G_{0}} \chi(\operatorname{det} \Phi+\text { H.c. }) \\
& =g_{\chi} \frac{G}{G_{0}} \chi\left(\sigma^{2}+\vec{\pi}^{2}-\eta^{2}-\vec{a}_{0}^{2}\right),
\end{aligned}
$$

where we abbreviated $g_{\chi}^{(1)} \equiv-2 g_{\chi}$ and only considered $T_{33} \equiv$ $\chi$ because this is the only four-quark state that exists for two flavors. This term is similar to the determinant term in Eq. (8), which models the $\mathrm{U}(1)_{\mathrm{A}}$ anomaly. Since the chiral condensate also induces a condensate of the scalar four-quark state, Eq. (20) generates a contribution to the masses of $\sigma$ and $\pi$ and, of the same magnitude but with opposite sign, to those of $\eta$ and $a_{0}$.

Very similar terms are obtained for the coupling to (axial-) vector quarkonia:

$$
\begin{aligned}
\mathcal{L}_{\mathrm{T}-\mathrm{AV}}= & g_{\mathrm{AV}}^{(1)} \frac{G}{G_{0}} \operatorname{Tr}\left[D_{\mathrm{R}} R_{\mu}^{\mathrm{T}} D_{\mathrm{R}}^{\dagger} R^{\mu}+D_{\mathrm{L}} L_{\mu}^{\mathrm{T}} D_{\mathrm{L}}^{\dagger} L^{\mu}\right] \\
& +g_{\mathrm{AV}}^{(2)} \frac{G}{G_{0}} \operatorname{Tr}\left[D_{\mathrm{R}} D_{\mathrm{R}}^{\dagger} R_{\mu}^{\dagger} R^{\mu}+D_{\mathrm{L}} D_{\mathrm{L}}^{\dagger} L_{\mu}^{\dagger} L^{\mu}\right] \\
& +g_{\mathrm{AV}}^{(3)} \frac{G}{G_{0}} \operatorname{Tr}\left[D_{\mathrm{R}} D_{\mathrm{R}}^{\dagger}+D_{\mathrm{L}} D_{\mathrm{L}}^{\dagger}\right] \operatorname{Tr}\left[R_{\mu}^{\dagger} R^{\mu}+L_{\mu}^{\dagger} L^{\mu}\right] .
\end{aligned}
$$

Again, we are only interested in the leading-order term at large $N_{\text {c }}$. Neglecting the pseudoscalar diquark $\tilde{D}$, we obtain

$$
\begin{aligned}
\mathcal{L}_{\mathrm{T}-\mathrm{AV}} & =\frac{g_{\mathrm{AV}}^{(1)}}{2} \frac{G}{G_{0}} \operatorname{Tr}\left[D R_{\mu}^{\mathrm{T}} D^{\dagger} R^{\mu}+D L_{\mu}^{\mathrm{T}} D^{\dagger} L^{\mu}\right] \\
& =\frac{g_{\mathrm{AV}}^{(1)}}{2} \frac{G}{G_{0}} T_{a b} \operatorname{Tr}\left[A_{b} R_{\mu}^{\mathrm{T}} A_{a}^{\mathrm{T}} R^{\mu}+A_{b} L_{\mu}^{\mathrm{T}} A_{a}^{\mathrm{T}} L^{\mu}\right] .
\end{aligned}
$$

\footnotetext{
${ }^{1}$ We note that this term is precisely the same as the one in Eq. (16) of Ref. [87]. However, in that work the interaction term is constructed by using the four-quark matrix analog of $\Phi$, which transforms in the same manner as $\Phi$ except under $\mathrm{U}(1)_{\mathrm{A}}$ transformations. Although both approaches yield the same interaction term as above, the other two interaction terms of Eq. (19) are only found using our approach.
} 
For two flavors, this term reduces to

$$
\begin{aligned}
\mathcal{L}_{\mathrm{T}-\mathrm{AV}} & =-2 g_{\mathrm{AV}} \frac{G}{G_{0}} \chi\left(\operatorname{det} R_{\mu}+\operatorname{det} L_{\mu}\right) \\
& =g_{\mathrm{AV}} \frac{G}{G_{0}} \chi\left(\vec{\rho}_{\mu}^{2}+\vec{a}_{1, \mu}^{2}-\omega_{\mu}^{2}-f_{1, \mu}^{2}\right),
\end{aligned}
$$

where we abbreviated $g_{\mathrm{AV}}^{(1)} \equiv-2 g_{\mathrm{AV}}$. Interestingly, this term looks structurally similar to Eq. (21). It generates a contribution to the masses of the isoscalar (axial-)vector mesons and, of the same magnitude but with opposite sign, to those of the isovector (axial-)vector mesons.

Introducing also a kinetic and mass term for the scalar tetraquark, we find the complete two-flavor four-quark
Lagrangian to be

$$
\begin{aligned}
\mathcal{L}_{\chi \text {-int }}= & \frac{1}{2} \partial_{\mu} \chi \partial^{\mu} \chi-\frac{1}{2} m_{\chi}^{2} \frac{G^{2}}{G_{0}^{2}} \chi^{2} \\
& +g_{\chi} \frac{G}{G_{0}} \chi\left(\sigma^{2}+\vec{\pi}^{2}-\eta^{2}-\vec{a}_{0}^{2}\right) \\
& +g_{\mathrm{AV}} \frac{G}{G_{0}} \chi\left(\vec{\rho}_{\mu}^{2}+\vec{a}_{1, \mu}^{2}-\omega_{\mu}^{2}-f_{1, \mu}^{2}\right) .
\end{aligned}
$$

From this Lagrangian and Eq. (8) we can derive masses and decay widths as well as the pion-pion scattering lengths.

\section{Masses of the scalar-isoscalar resonances}

The terms in the Lagrangian which, upon condensation of $\sigma, G$, and $\chi$, give rise to the mass matrix for the scalarisoscalar resonances are

$$
-\frac{1}{2} \mu^{2} \frac{G^{2}}{G_{0}^{2}} \sigma^{2}+\frac{c}{2} \sigma^{2}-\frac{1}{4}\left(\lambda_{1}+\frac{\lambda_{2}}{2}\right) \sigma^{4}-\frac{1}{2} m_{\chi}^{2} \frac{G^{2}}{G_{0}^{2}} \chi^{2}+g_{\chi} \frac{G}{G_{0}} \chi \sigma^{2}-\frac{1}{4} \frac{m_{G}^{2}}{\Lambda_{\mathrm{dil}}^{2}} G^{4}\left(\ln \left|\frac{G}{\Lambda_{\mathrm{dil}}}\right|-\frac{1}{4}\right) .
$$

We perform a shift of the scalar-isoscalar fields by their respective vacuum expectation values, $\sigma \rightarrow \varphi+\sigma, G \rightarrow G_{0}+G$, $\chi \rightarrow \chi_{0}+\chi$. This leads to mass terms for these three fields, which can be compactly written in matrix form as

$$
V_{\text {mass }}(\chi, \sigma, G)=\frac{1}{2}(\chi, \sigma, G)\left(\begin{array}{ccc}
m_{\chi}^{2} & -2 g_{\chi} \varphi & g_{\chi} \frac{\varphi^{2}}{G_{0}} \\
-2 g_{\chi} \varphi & m_{\sigma}^{2} & \frac{2 \varphi}{G_{0}}\left(\mu^{2}-g_{\chi} \chi_{0}\right) \\
g_{\chi} \frac{\varphi^{2}}{G_{0}} & \frac{2 \varphi}{G_{0}}\left(\mu^{2}-g_{\chi} \chi_{0}\right) & M_{G}^{2}
\end{array}\right)\left(\begin{array}{l}
\chi \\
\sigma \\
G
\end{array}\right) \equiv \frac{1}{2}(\chi, \sigma, G) M\left(\begin{array}{l}
\chi \\
\sigma \\
G
\end{array}\right),
$$

where $m_{\sigma}^{2}$ is given in Eq. (B4) and

$$
M_{G}^{2}=\frac{\mu^{2} \varphi^{2}+m_{\chi}^{2} \chi_{0}^{2}}{G_{0}^{2}}+m_{G}^{2} \frac{G_{0}^{2}}{\Lambda_{\mathrm{dil}}^{2}}\left(1+3 \ln \left|\frac{G_{0}}{\Lambda_{\mathrm{dil}}}\right|\right) .
$$

Note that the (13) and (31) elements of $M$ were simplified using the condition that $\chi_{0}$ is an extremum of the potential energy density. The real, symmetric mass matrix $M$ can be diagonalized by an orthogonal transformation $O, O^{T} M O=$ $M_{\text {diag }}$ :

$$
M_{\text {diag }}=\left(\begin{array}{ccc}
M_{H}^{2} & 0 & 0 \\
0 & M_{S}^{2} & 0 \\
0 & 0 & M_{G^{\prime}}^{2}
\end{array}\right),
$$

where the eigenvalues $M_{H}^{2}, M_{S}^{2}$, and $M_{G^{\prime}}^{2}$ correspond to the (squared) masses of the physical fields $f_{0}(500), f_{0}(1370)$, and $f_{0}(1710)$. These fields are linear combinations of the unphysical fields $\chi, \sigma$, and $G$, given by

$$
\left(\begin{array}{c}
f_{0}(500) \\
f_{0}(1370) \\
f_{0}(1710)
\end{array}\right)=\left(\begin{array}{c}
H \\
S \\
G^{\prime}
\end{array}\right)=O^{T}\left(\begin{array}{c}
\chi \\
\sigma \\
G
\end{array}\right)
$$

\section{Baryons}

Baryons are implemented in the eLSM in the so-called mirror assignment $[35,37,73]$. One introduces two baryon doublets, $\Psi_{1}$ and $\Psi_{2}$, where $\Psi_{1}$ has positive parity and $\Psi_{2}$ is its chiral partner with negative parity. In the mirror assignment these fields transform under chiral transformations as

$$
\Psi_{1, \mathrm{R} / \mathrm{L}} \rightarrow U_{\mathrm{R} / \mathrm{L}} \Psi_{1, \mathrm{R} / \mathrm{L}}, \quad \Psi_{2, \mathrm{R} / \mathrm{L}} \rightarrow U_{\mathrm{L} / \mathrm{R}} \Psi_{2, \mathrm{R} / \mathrm{L}},
$$

i.e., $\Psi_{1, \mathrm{R} / \mathrm{L}}$ transforms like a vector under $\mathrm{SU}\left(N_{\mathrm{f}}\right)_{\mathrm{R} / \mathrm{L}}$, as expected, while $\Psi_{2, \mathrm{R} / \mathrm{L}}$ transforms in a mirror way, like a vector under $\mathrm{SU}\left(N_{\mathrm{f}}\right)_{\mathrm{L} / \mathrm{R}}$. Both fields are singlets under $\mathrm{U}(1)_{\mathrm{A}}$ transformations.

The mirror assignment allows for the existence of a new chirally invariant mass term, which contributes to the baryon masses in a different manner than the chiral condensate. Thus, baryons can have nonzero masses even when the chiral condensate vanishes. Demanding dilatation invariance, the new mass term must necessarily arise from coupling the baryons to the four-quark field $\chi$,

$$
-a \chi\left(\bar{\Psi}_{1 \mathrm{~L}} \Psi_{2 \mathrm{R}}-\bar{\Psi}_{1 \mathrm{R}} \Psi_{2 \mathrm{~L}}+\text { H.c. }\right),
$$

and/or the glueball field $G$,

$$
-b G\left(\bar{\Psi}_{1 \mathrm{~L}} \Psi_{2 \mathrm{R}}-\bar{\Psi}_{1 \mathrm{R}} \Psi_{2 \mathrm{~L}}+\text { H.c. }\right),
$$

and subsequent condensation of $\chi$ and $G$. Both $G$ and $\chi$ are chiral singlets, as shown in Appendix A.

Furthermore, it is possible to introduce another interaction term that violates $\mathrm{U}(1)_{\mathrm{A}}$ symmetry but gives a contribution to the nucleon mass as well. This term is given by

$$
\begin{aligned}
\mathcal{L}_{\text {anom. }}= & -c_{N}(\operatorname{det} \Phi+\text { H.c. })\left(\bar{\Psi}_{1 \mathrm{~L}} \Psi_{2 \mathrm{R}}-\bar{\Psi}_{1 \mathrm{R}} \Psi_{2 \mathrm{~L}}+\text { H.c. }\right) \\
= & -\frac{c_{N}}{2}\left(\sigma^{2}+\vec{\pi}^{2}-\eta^{2}-\vec{a}_{0}^{2}\right) \\
& \times\left(\bar{\Psi}_{1 \mathrm{~L}} \Psi_{2 \mathrm{R}}-\bar{\Psi}_{1 \mathrm{R}} \Psi_{2 \mathrm{~L}}+\text { H.c. }\right) .
\end{aligned}
$$


Such an anomalous term (which also breaks the dilatation symmetry) yields a four-point vertex that has not been considered before in this model; see also the last diagram of Fig. 2 (found in Appendix E).

The terms in the baryon Lagrangian which are relevant for pion-nucleon scattering are then

$$
\begin{aligned}
\mathcal{L}_{\mathrm{eLSM}}^{\mathrm{bar}}= & \bar{\Psi}_{1 \mathrm{~L}} i \gamma_{\mu} D_{1 \mathrm{~L}}^{\mu} \Psi_{1 \mathrm{~L}}+\bar{\Psi}_{1 \mathrm{R}} i \gamma_{\mu} D_{1 \mathrm{R}}^{\mu} \Psi_{1 \mathrm{R}}+\bar{\Psi}_{2 \mathrm{~L}} i \gamma_{\mu} D_{2 \mathrm{R}}^{\mu} \Psi_{2 \mathrm{~L}} \\
& +\bar{\Psi}_{2 \mathrm{R}} i \gamma_{\mu} D_{2 \mathrm{~L}}^{\mu} \Psi_{2 \mathrm{R}}-\hat{g}_{1}\left(\bar{\Psi}_{1 \mathrm{~L}} \Phi \Psi_{1 \mathrm{R}}+\text { H.c. }\right) \\
& -\hat{g}_{2}\left(\bar{\Psi}_{2 \mathrm{~L}} \Phi^{\dagger} \Psi_{2 \mathrm{R}}+\text { H.c. }\right) \\
& -\left[a \chi+b G+\frac{c_{N}}{2}\left(\sigma^{2}+\vec{\pi}^{2}\right)\right] \\
& \times\left(\bar{\Psi}_{1 \mathrm{~L}} \Psi_{2 \mathrm{R}}-\bar{\Psi}_{1 \mathrm{R}} \Psi_{2 \mathrm{~L}}+\text { H.c. }\right)+\cdots
\end{aligned}
$$

where $D_{1 / 2, \mathrm{R}}^{\mu}=\partial^{\mu}-i c_{1 / 2} R^{\mu}, D_{1 / 2, \mathrm{~L}}^{\mu}=\partial^{\mu}-i c_{1 / 2} L^{\mu}$.

Upon condensation of $\chi, G$, and $\sigma$ a baryonic mass term is generated:

$$
m_{0} \equiv a \chi_{0}+b G_{0}+\frac{c_{N} \varphi^{2}}{2} .
$$

The mass term mixes $\Psi_{1}$ and $\Psi_{2}$, so that the physical fields are obtained by a unitary transformation,

$$
\left(\begin{array}{c}
N \\
N^{*}
\end{array}\right)=\frac{1}{\sqrt{2 \cosh \delta}}\left(\begin{array}{cc}
e^{\delta / 2} & \gamma_{5} e^{-\delta / 2} \\
\gamma_{5} e^{-\delta / 2} & -e^{\delta / 2}
\end{array}\right)\left(\begin{array}{l}
\Psi_{1} \\
\Psi_{2}
\end{array}\right)
$$

where $\delta$ is the mixing parameter.

We recall that the quantity $m_{0}$ does not represent the baryon mass in the chiral limit (i.e., when the bare quark masses are set to zero), but represents the chirally invariant contribution to the nucleon mass, which is the same for both the nucleon $N(939)$ and its chiral partner $N(1535)$. As Eq. (36) shows, in our case $m_{0}$ consists of three contributions: the scalar tetraquark condensate $\chi_{0}$, the dilaton condensate $G_{0}$, and also the chiral condensate $\varphi$. The difference in mass between $N$ and $N^{*}(1535)$ is, in contrast, solely proportional to the chiral condensate $\varphi$. If we consider the limit $\varphi \rightarrow 0$ (no SSB) one obtains $m_{0} \rightarrow b G_{0} \neq 0$, hence without SSB the nucleon and its chiral partner would have an identical nonzero mass. (Note that $\chi_{0}$ vanishes also when $\varphi \rightarrow 0$ since it is proportional to $\left.\varphi^{2}[71]\right)$.

\section{RESULTS}

In this section we first perform a global fit of the parameters in the meson sector. Here we consider two different scenarios: first we neglect the scalar glueball and investigate the mixing of the scalar four-quark with the quarkonium state only. Then, we present the results for the full three-scalar mixing problem, which includes the scalar glueball, the four-quark, and the quarkonium state. This allows us to estimate the importance of the scalar glueball for the calculation of the decay widths of the scalar-isoscalars and the pion-pion scattering lengths. Subsequently, we will take the results from the global fit of the meson sector and calculate pion-nucleon scattering parameters.
TABLE I. The masses of the fields as given by the PDG [1].

\begin{tabular}{lcc}
\hline \hline Field & Assignment & Masses \\
\hline$H$ & $f_{0}(500)$ & $475 \pm 75 \mathrm{MeV}$ \\
$S$ & $f_{0}(1370)$ & $1350 \pm 150 \mathrm{MeV}$ \\
$G^{\prime}$ & $f_{0}(1710)$ & $1723 \pm 5 \mathrm{MeV}$ \\
$a_{0}$ & $a_{0}(1450)$ & $1474 \pm 19 \mathrm{MeV}$ \\
$a_{1}$ & $a_{1}(1260)$ & $1230 \pm 40 \mathrm{MeV}$ \\
$\rho$ & $\rho(770)$ & $775.26 \pm 0.25 \mathrm{MeV}$ \\
$f_{1}$ & $f_{1}(1285)$ & $1281.9 \pm 0.5 \mathrm{MeV}$ \\
$\omega$ & $\omega(782)$ & $782.65 \pm 0.12 \mathrm{MeV}$ \\
\hline \hline
\end{tabular}

\section{A. Global fit in the meson sector}

The assignment of our effective hadronic degrees of freedom is given in Table I. For this assignment the experimental data for the decay widths and the pion-pion scattering lengths are given in Table II. For the mass of the pion we use the isospin-averaged mass, $m_{\pi}=138 \mathrm{MeV}$. For the mass of $a_{1}$ we choose $1277 \mathrm{MeV}$, which is slightly above the upper error band of the Particle Data Group (PDG) data. The reason for this choice is the mass splitting between the isoscalar and the isovector (axial-)vector mesons generated by the coupling between the four-quark state and the (axial-)vector mesons in Eq. (24),

$$
m_{\rho}^{2}-m_{\omega}^{2}=m_{a_{1}}^{2}-m_{f_{1}}^{2}=4 g_{\mathrm{AV}} \chi_{0} ;
$$

see also Appendices B 2 and B 3. Since the masses of $\rho, \omega$, and $f_{1}$ are known to very good precision (see Table I), we are forced to increase the theoretical value for the mass of $a_{1}$ such that the mass splitting between that state and $f_{1}$ is of the same order as that between $\rho$ and $\omega$.

Furthermore, the physical $\eta$ meson contains a considerable $\bar{s} s$ admixture, which, in a pure two-flavor scenario, has to be eliminated by a rotation in the $\eta-\eta^{\prime}$ sector. The result is the value $m_{\eta}=755 \mathrm{MeV}$ for the mass of the purely nonstrange $\eta$ meson [28].

The pion-pion scattering parameters in the eLSM were first calculated in Ref. [27], but without a scalar four-quark state and without a dynamical scalar glueball. It was found that the pion-pion scattering length $m_{\pi} a_{0}^{0}$ is in the range of experimental data only for a small mass of the scalar-isoscalar quarkonium $\sigma$ field, while $m_{\pi} a_{0}^{2}$ agrees well with experimental data for all values of the $\sigma$ mass (cf. Fig. 2 in Ref. [27]). Here we examine how the results change if we consider a

TABLE II. The decay widths $\Gamma_{H \rightarrow \pi \pi}$ and $\Gamma_{G^{\prime} \rightarrow \pi \pi}$ as given by the PDG [1], the decay width $\Gamma_{S \rightarrow \pi \pi}$ is taken from Ref. [94], and the scattering lengths from Ref. [95].

\begin{tabular}{lc}
\hline \hline Observable & Experimental data \\
\hline$\Gamma_{H \rightarrow \pi \pi}$ & $550 \pm 150 \mathrm{MeV}$ \\
$\Gamma_{S \rightarrow \pi \pi}$ & $350 \pm 150 \mathrm{MeV}$ \\
$\Gamma_{G^{\prime} \rightarrow \pi \pi}$ & $29.3 \pm 6.5 \mathrm{MeV}$ \\
$m_{\pi} a_{0}^{0}$ & $0.218 \pm 0.02$ \\
$m_{\pi} a_{0}^{2}$ & $-0.046 \pm 0.013$ \\
\hline \hline
\end{tabular}


TABLE III. In the upper left box the fitted parameters are given. The parameters in the lower left box are calculated from the fitted parameters. $\theta$ is the mixing angle between $\chi$ and $\sigma$. In the right box the numerical results for the observables are given.

\begin{tabular}{lccc}
\hline \hline Param. & Value & Observ. & Value \\
\hline$g_{\chi}$ & $2.86 \pm 0.53 \mathrm{MeV}$ & $M_{H}$ & $533 \pm 33 \mathrm{MeV}$ \\
$h$ & $-0.22 \pm 4.7$ & $M_{S}$ & $1405 \mathrm{MeV}$ \\
$m_{\chi}$ & $533 \pm 33 \mathrm{MeV}$ & $\Gamma_{H \rightarrow \pi \pi}$ & $504 \pm 148 \mathrm{MeV}$ \\
$g_{\mathrm{AV}}$ & $-12018 \pm 1365 \mathrm{MeV}$ & $\Gamma_{S \rightarrow \pi \pi}$ & $420 \pm 144 \mathrm{MeV}$ \\
$\mu^{2}$ & $-879 \times 10^{3} \mathrm{MeV}^{2}$ & $m_{\pi} a_{0}^{0}$ & $0.210 \pm 0.016$ \\
$m_{1}^{2}$ & $\approx 775^{2} \mathrm{MeV}^{2}$ & $m_{\pi} a_{0}^{2}$ & $-0.027 \pm 0.005$ \\
$c$ & $99 \pm 0.4 \times 10^{3} \mathrm{MeV}^{2}$ & & \\
$m_{\sigma}$ & $1405 \mathrm{MeV}^{2}$ & $\chi^{2}$ test & Value \\
\cline { 2 - 4 }$\chi_{0}$ & $0.24 \pm 0.02 \mathrm{MeV}$ & $\chi^{2}$ & 3.5 \\
$\theta$ & $\approx 0$ & $\chi_{\text {red }}^{2}$ & 1.8 \\
\hline \hline
\end{tabular}

scalar four-quark state $\chi$ in addition to the quarkonium state $\sigma$. At first, we neglect the scalar glueball.

The Lagrangians (8) and (25) contain ten parameters that are of relevance for our fit: $\mu^{2}, \lambda_{1}, \lambda_{2}, c, m_{1}^{2}, h_{1}+h_{2} \equiv$ $h, h_{3}, g_{\chi}, g_{\mathrm{AV}}, m_{\chi}$. The parameters $\lambda_{2}, h_{3}, c, \mu^{2}, m_{1}^{2}, g_{\mathrm{AV}}$ can be expressed by the physical masses of Table I or by the remaining model parameters; see Appendix B. Furthermore, $\lambda_{1}$ is large- $N_{c}$ suppressed and is therefore set to zero. Thus, only the three parameters $h, g_{\chi}, m_{\chi}$ need to be fitted. We used the standard $\chi^{2}$ procedure to fit the parameters and determine the errors $\left[\chi^{2}=\chi^{2}\left(h, g_{\chi}, m_{\chi}\right)\right]$ :

$$
\begin{aligned}
\chi^{2}= & \left(\frac{M_{H}-475 \mathrm{MeV}}{75 \mathrm{MeV}}\right)^{2}+\left(\frac{M_{S}-1350 \mathrm{MeV}}{150 \mathrm{MeV}}\right)^{2} \\
& +\left(\frac{\Gamma_{H \rightarrow \pi \pi}-550 \mathrm{MeV}}{150 \mathrm{MeV}}\right)^{2}+\left(\frac{\Gamma_{S \rightarrow \pi \pi}-350 \mathrm{MeV}}{150 \mathrm{MeV}}\right)^{2} \\
& +\left(\frac{m_{\pi} a_{0}^{0}-0.218}{0.02}\right)^{2}+\left(\frac{m_{\pi} a_{0}^{2}+0.046}{0.013}\right)^{2}
\end{aligned}
$$

The result of this fit is presented in Table III.

Next, we consider the scalar glueball as dynamical field as well. Now, $M_{G}$ and $G_{0}$ are additional fit parameters. Then the $\chi^{2}$ function is given as

$$
\begin{aligned}
\chi^{2}= & \left(\frac{M_{H}-475 \mathrm{MeV}}{75 \mathrm{MeV}}\right)^{2}+\left(\frac{M_{S}-1350 \mathrm{MeV}}{150 \mathrm{MeV}}\right)^{2} \\
& +\left(\frac{m_{G^{\prime}}-1720 \mathrm{MeV}}{50 \mathrm{MeV}}\right)^{2}+\left(\frac{\Gamma_{H \rightarrow \pi \pi}-550 \mathrm{MeV}}{150 \mathrm{MeV}}\right)^{2} \\
& +\left(\frac{\Gamma_{S \rightarrow \pi \pi}-350 \mathrm{MeV}}{150 \mathrm{MeV}}\right)^{2}+\left(\frac{\Gamma_{G^{\prime} \rightarrow \pi \pi}-29.3 \mathrm{MeV}}{6.5 \mathrm{MeV}}\right)^{2} \\
& +\left(\frac{m_{\pi} a_{0}^{0}-0.218}{0.02}\right)^{2}+\left(\frac{m_{\pi} a_{0}^{2}+0.046}{0.013}\right)^{2} .
\end{aligned}
$$

The results of the fit for this scenario are given in Table IV.
TABLE IV. The result of the fit where the glueball is included.

\begin{tabular}{lccc}
\hline \hline Param. & Value & Observ. & Value \\
\hline$g_{\chi}$ & $3.06 \pm 0.54 \mathrm{MeV}$ & $M_{H}$ & $546 \pm 33 \mathrm{MeV}$ \\
$h$ & $5.53 \pm 2.75$ & $M_{S}$ & $1238 \pm 113 \mathrm{MeV}$ \\
$M_{G}$ & $1564 \pm 84 \mathrm{MeV}$ & $M_{G^{\prime}}$ & $1696 \pm 49 \mathrm{MeV}$ \\
$G_{0}$ & $428 \pm 135 \mathrm{MeV}$ & $\Gamma_{H \rightarrow \pi \pi}$ & $539 \pm 148 \mathrm{MeV}$ \\
$m_{\chi}$ & $547 \pm 33 \mathrm{MeV}$ & $\Gamma_{S \rightarrow \pi \pi}$ & $503 \pm 98 \mathrm{MeV}$ \\
$g_{\mathrm{AV}}$ & $-11820 \pm 738 \mathrm{MeV}$ & $\Gamma_{G^{\prime} \rightarrow \pi \pi}$ & $29 \pm 7 \mathrm{MeV}$ \\
$\mu^{2}$ & $-873 \times 10^{3} \mathrm{MeV}^{2}$ & $m_{\pi} a_{0}^{0}$ & $0.210 \pm 0.016$ \\
$m_{1}^{2}$ & $730^{2} \mathrm{MeV}^{2}$ & $m_{\pi} a_{0}^{2}$ & $-0.028 \pm 0.005$ \\
$c$ & $99 \times 10^{3} \mathrm{MeV}^{2}$ & $\chi^{2}$ test & Value \\
\cline { 2 - 4 }$m_{\sigma}$ & $1401 \mathrm{MeV}^{2}$ & $\chi^{2}$ & 5.1 \\
$\chi_{0}$ & $0.24 \pm 0.02 \mathrm{MeV}$ & $\chi_{\text {red }}^{2}$ & 1.7 \\
\hline \hline
\end{tabular}

From this fit the following scalar-isoscalar mixing matrix is obtained,

$$
O^{T}=\left(\begin{array}{ccc}
1.00 & 0.00 & 0.00 \\
0.00 & 0.81 & -0.59 \\
0.00 & 0.59 & 0.81
\end{array}\right)
$$

which corresponds to the following admixtures of the physical states:

$$
\begin{aligned}
& f_{0}(500): \quad 100 \% \chi, \quad 0 \% \sigma, \quad 0 \% G, \\
& f_{0}(1370): 0 \% \chi, \quad 65 \% \sigma, \quad 35 \% G \text {, } \\
& f_{0}(1710): \quad 0 \% \chi, \quad 35 \% \sigma, \quad 65 \% G \text {. }
\end{aligned}
$$

Let us briefly discuss these results:

(1) Our aim was to correctly reproduce the masses and decay widths of $f_{0}(500), f_{0}(1370)$, and $f_{0}(1710)$. The fit agrees well with experimental data, only for the scattering length $m_{\pi} a_{0}^{2}$ the theoretical and experimental error bands overlap just barely (the theoretical value is slightly too large). In both fits, with and without a dynamical glueball, similar parameters are obtained, which results in very similar observables.

(2) The parameter determining the mixing of the four-quark state with the quarkonium state is $-2 g_{\chi} \varphi /\left(m_{\sigma}^{2}-m_{\chi}^{2}\right)$, which is (approximately) zero, explaining why $f_{0}(500)$ is (almost to) $100 \%$ a fourquark state.

(3) Although the value of $g_{\chi}$ is very small, it is numerically not negligible. In Appendix B we show that $g_{\mathrm{AV}}$ is proportional to the inverse of $g_{\chi}$. Thus, $g_{\mathrm{AV}}$ would diverge if we send $g_{\chi} \rightarrow 0$.

(4) Since $g_{\chi}$ is very small, the coupling $g_{\mathrm{AV}}$ between the scalar four-quark state and the (axial-)vector mesons is rather large, $g_{\mathrm{AV}} \approx-12 \mathrm{GeV}$ in both fits.

(5) We also tried to identify $H=f_{0}(980)$, another possible candidate for a four-quark state, but no reasonable fit results were obtained. Our investigation clearly favors the (nonstrange) scalar four-quark state to be a light and broad state.

(6) The value of $\Gamma_{S \rightarrow \pi \pi}$ used in our fit is somewhat problematic due to its uncertain value in the literature [1,94]. If we exclude this width from our fit, we 
TABLE V. Parameters determined by a fit of $g_{A}^{N}, g_{A}^{N^{*}}, \Gamma_{N^{*} \rightarrow N \pi}, \Gamma_{a_{1} \rightarrow \pi \gamma}$, where $N^{*}$ is assigned to $N(1535)$; see Ref. [35].

\begin{tabular}{lc}
\hline \hline Parameter & Value \\
\hline$c_{1}$ & $-3.0 \pm 0.6$ \\
$c_{2}$ & $11.6 \pm 3.6$ \\
$Z$ & $1.67 \pm 0.2$ \\
$m_{0}$ & $462 \pm 136 \mathrm{MeV}$ \\
\hline \hline
\end{tabular}

obtain $\Gamma_{S \rightarrow \pi \pi} \approx 3 \mathrm{GeV}$ for the case without dynamical glueball and $\Gamma_{S \rightarrow \pi \pi} \approx 750 \mathrm{MeV}$ for the case with dynamical glueball, while the other observables change only slightly.

(7) We obtain a fit of similar quality if we assign $G^{\prime}=$ $f_{0}(1500)$, but at a cost of a very large dilaton condensate $G_{0}>1.5 \mathrm{GeV}$. Thus, we cannot make any prediction about whether $f_{0}(1500)$ or $f_{0}(1710)$ is more likely the glueball candidate. This question has been addressed in a model similar to ours [30], where the authors found $f_{0}(1710)$ to be the scalar glueball while $f_{0}(1500)$ was found to be mostly an $\bar{s} s$ quarkonium state.

(8) The elements of the matrix (41) which correspond to the mixing between $\sigma$ and $G$ are somewhat larger than those of Ref. [30], most likely due to the missing strange scalar-isoscalar $\sigma_{S}$ in our two-flavor model.

(9) We find in both fits very similar values for the pion-pion scattering lengths, indicating that the scalar glueball is actually not important for pion-pion scattering, which is not too surprising because of its large mass.

(10) We checked that the pion-pion scattering lengths vanish in the chiral limit, i.e., $m_{\pi} \rightarrow 0$, as required by low-energy theorems.

(11) To further underline the importance of a light scalarisoscalar resonance we can take the limit $g_{\chi} \rightarrow 0$ and $g_{\mathrm{AV}} \rightarrow 0$ to turn off the interactions of the four-quark state. Performing a fit in this limit leads to $m_{\pi} a_{0}^{0}=$ 0.156 and $m_{\pi} a_{0}^{2}=-0.044$, i.e., results comparable to those of Ref. [27] for large $\sigma$ masses. This result is obtained for both cases, with and without a dynamical scalar glueball.

\section{B. Pion-nucleon scattering parameters}

Some of the parameters of the baryon Lagrangian have been already determined in Ref. [35] and are reported in Table V.

In Ref. [35] the isospin-even and isospin-odd scattering lengths have been calculated in a model without a scalar fourquark state and a scalar glueball. The authors found $m_{\pi} a_{0}^{(-)}=$ $0.0834 \pm 0.0087$ for the isospin-odd scattering length, which is in surprisingly good agreement with the experimental value, see the first entry of the last column in Table VI. However, there were several errors in Eq. (19) of Ref. [35]. The correct formula is given in Appendix E, and the correct value is the first entry of the second column in Table VI. This value is now outside the experimental error band.
TABLE VI. Isospin-odd scattering parameters.

\begin{tabular}{lcc}
\hline \hline Parameter & Value & Experiment \\
\hline$m_{\pi} a_{0}^{(-)}$ & 0.0782 & $0.0861 \pm 0.0009$ \\
$m_{\pi}^{3} a_{1+}^{(-)}$ & -0.048 & $-0.081 \pm 0.002$ \\
$m_{\pi}^{3} a_{1-}^{(-)}$ & -0.042 & $-0.013 \pm 0.003$ \\
$m_{\pi}^{3} r_{0}^{(-)}$ & 0.022 & $0.007 \pm 0.005$ \\
\hline \hline
\end{tabular}

As experimental inputs for the scattering lengths, we use the results of the analysis of Refs. [96,97], which are based on experimental results (see, e.g., $[98,99]$ ) and isolate the contributions of isospin-breaking electromagnetic interactions (which are not present in our model). The remaining scattering parameters are taken from Ref. [100].

The isospin-even scattering length $m_{\pi} a_{0}^{(+)}$has also been calculated in Ref. [35]. There was also a sign error in Eq. (18) of Ref. [35], which changes the behavior of $m_{\pi} a_{0}^{(+)}$as a function of the parameter $m_{1}$ as shown in Fig. 2 of Ref. [35]. The correct formula is also given in Appendix E. We do not show the corrected graphs; in the first entry of the second column in Table VII we simply list the corresponding value for $m_{1}=643 \mathrm{MeV}$ obtained from the global fit of Ref. [28]. The theoretical value of the scattering length has the opposite sign compared to the experimental value.

In addition, here we also calculate isospin-even and isospin-odd scattering volumes and effective range parameters within the setup of Ref. [35]. The corresponding values are shown in the second to fourth rows in Tables VI and VII. The isospin-odd scattering volumes and the range parameter deviate by factors of 0.5 to 3 from the experimental values, the isospin-even scattering volumes by factors of 0.4 to 2 , while the isospin-even range parameter is about a factor 7 too small. Note that all scattering parameters have also theoretical errors originating from the uncertainties of the $\chi^{2}$ fit in determining the parameters. However, we omitted the errors because they are smaller than $5 \%$ and therefore not a reliable measure of uncertainty.

The theoretical values in Tables VI and VII were computed without a scalar four-quark state or scalar glueball, i.e., they are just a correction and extension of the results of Ref. [35]. While the isospin-odd scattering parameters are influenced neither by a scalar four-quark state nor by a scalar glueball, and thus cannot be further improved within our model, we can still study the question whether the introduction of these states can at least improve the description of the isospin-even

TABLE VII. Isospin-even scattering parameters for $m_{1}=$ $643 \mathrm{MeV}$ and $m_{\sigma}=1370 \mathrm{MeV}$ as obtained in the model of Ref. [35] (shown are the corrected values).

\begin{tabular}{lcc}
\hline \hline Parameter & Value & Experiment \\
\hline$m_{\pi} a_{0}^{(+)}$ & -0.0083 & $0.0076 \pm 0.0031$ \\
$m_{\pi}^{3} a_{1+}^{(+)}$ & 0.049 & $0.130 \pm 0.003$ \\
$m_{\pi}^{3} a_{1-}^{(+)}$ & -0.093 & $-0.056 \pm 0.010$ \\
$m_{\pi}^{3} r_{0}^{(+)}$ & 0.009 & $-0.06 \pm 0.02$ \\
\hline \hline
\end{tabular}


TABLE VIII. Results with $a=1897.33, b=c_{N}=0$.

\begin{tabular}{lcc}
\hline \hline Parameter & Value & Experiment \\
\hline$m_{\pi} a_{0}^{(+)}$ & 11.196 & $0.0076 \pm 0.0031$ \\
$m_{\pi}^{3} a_{1+}^{(+)}$ & -3.422 & $0.133 \pm 0.004$ \\
$m_{\pi}^{3} a_{1-}^{(+)}$ & -3.365 & $-0.056 \pm 0.010$ \\
$m_{\pi}^{3} r_{0}^{(+)}$ & 9.061 & $-0.06 \pm 0.02$ \\
\hline \hline
\end{tabular}

scattering parameters. The explicit calculations of the pionnucleon scattering amplitudes and the isospin-even scattering parameters are deferred to Appendix E. Compared to the model of Ref. [35] we have three new couplings, $a, b$, and $c_{N}$, the values of which are only constrained by the linear combination (36), which should have the correct value of $m_{0}$ in order to reproduce the mass of the nucleon and its chiral partner. In a first step we consider only one parameter at a time, while setting the other ones to zero. In this way we distinguish three cases:

$$
\begin{aligned}
& \text { A: } \quad a= \frac{m_{0}}{\chi_{0}}=1897.33, b=c_{N}=0, \\
& \text { B: } \quad b=\frac{m_{0}}{G_{0}}=1.078, a=c_{N}=0, \\
& \text { C: } \quad c_{N}=2 \frac{m_{0}}{\varphi^{2}}=\frac{0.0388}{\mathrm{MeV}}, a=b=0 .
\end{aligned}
$$

In Table VIII we consider case A, where only the scalar four-quark state contributes to the explicit mass term. In this case the scalar glueball contributes only indirectly to the pionnucleon scattering parameters via the $G-\sigma$ and $G-\chi$ mixing. Due to the small value of the four-quark condensate found in the fit of the meson sector the coupling between the scalar four-quark state and the nucleons must be extremely large in order to obtain $m_{0}=462 \mathrm{MeV}$. This leads to scattering parameters that are off by several orders of magnitude.

In Tables IX and X we consider the cases B and C, where $m_{0}$ originates either exclusively from the gluon condensate or from the anomaly contribution, respectively. In these cases, the scalar four-quark state contributes to pion-nucleon scattering only via the $\chi-\sigma$ and $\chi-G$ coupling. The results are rather similar for both cases, and the numerical values rather close to those of the model of Ref. [35]; cf. Table VII. This is expected because on the one hand the glueball is rather heavy and thus cannot substantially influence the scattering parameters in case B. On the other hand, the additional anomalous contribution $\sim c_{N} \varphi \simeq 6$ to the $\sigma N N^{*}$ coupling is about a factor of 2 smaller in magnitude than the Yukawa coupling $\left(\hat{g}_{1} e^{\delta}-\hat{g}_{2} e^{-\delta}\right) / 4$, and thus case $\mathrm{C}$ is, as far as pion-nucleon

TABLE IX. Results with $b=1.078, a=c_{N}=0$.

\begin{tabular}{lcc}
\hline \hline Parameter & Value & Experiment \\
\hline$m_{\pi} a_{0}^{(+)}$ & -0.0079 & $0.0076 \pm 0.0031$ \\
$m_{\pi}^{3} a_{1+}^{(+)}$ & 0.048 & $0.133 \pm 0.004$ \\
$m_{\pi}^{3} a_{1-}^{(+)}$ & -0.091 & $-0.056 \pm 0.010$ \\
$m_{\pi}^{3} r_{0}^{(+)}$ & 0.009 & $-0.06 \pm 0.02$ \\
\hline \hline
\end{tabular}

TABLE X. Results with $c_{N}=0.0388 / \mathrm{MeV}, a=b=0$.

\begin{tabular}{lcc}
\hline \hline Parameter & Value & Experiment \\
\hline$m_{\pi} a_{0}^{(+)}$ & -0.0078 & $0.0076 \pm 0.0031$ \\
$m_{\pi}^{3} a_{1+}^{(+)}$ & 0.048 & $0.133 \pm 0.004$ \\
$m_{\pi}^{3} a_{1-}^{(+)}$ & -0.091 & $-0.056 \pm 0.010$ \\
$m_{\pi}^{3} r_{0}^{(+)}$ & 0.008 & $-0.06 \pm 0.02$ \\
\hline \hline
\end{tabular}

scattering is concerned, essentially identical to the model of Ref. [35]. Case A is markedly different from cases B and C because of the presence of the light four-quark field $f_{0}(500)$, which has a substantial impact on pion-nucleon scattering parameters.

We now perform a simultaneous $\chi^{2}$ fit for all three parameters $a, b$, and $c_{N}$, respecting the constraint (36). Using the constraint that $b$ is positive yields the results of Table XI. It should also be noted that we find basically the same results if we set either $b=0$ or $c_{N}=0$. It is important, however, that $a$ is nonvanishing. Although the agreement between the theoretically calculated scattering parameters and experimental data is now in general better, the values still deviate by factors of 1.5 to 3 (and the scattering length $a_{0}^{(+)}$and the range parameter have the wrong sign).

\section{SUMMARY AND DISCUSSION}

In this paper, we studied the influence of the light fourquark state $f_{0}(500)$ and the scalar glueball on pion-pion and pion-nucleon scattering in the framework of the eLSM for $N_{\mathrm{f}}=2$ flavors. We first performed a $\chi^{2}$ fit to properties in the mesonic sector. We found a physically acceptable minimum for which the resonance $f_{0}(500)$ is (almost) exclusively a fourquark state, $f_{0}(1370)$ predominantly a light quark-antiquark state, and $f_{0}(1710)$ predominantly a gluonic state. The masses and the decay widths of these resonances as well as pion-pion scattering lengths can be correctly described; in particular $a_{0}^{0}$ is strongly dependent on the additional attraction on account of $f_{0}(500)$, and the presence of the latter is necessary for a good description of the data. The resonance $f_{0}(1710)$ is quite heavy, hence it does not affect the results in a substantial way, but its presence is nevertheless important to stabilize the fit (due to smaller errors and a reasonable upper limit for the gluon condensate $G_{0}$ ). Another notable result is the observation that the (axial) vectors turn out to interact quite strongly with the scalar four-quark state $\left(g_{\mathrm{AV}} / G_{0} \approx-30\right)$, while the coupling to the (pseudo)scalar quarkonia is rather small $\left(g_{\chi} / G_{0} \approx 0\right)$.

TABLE XI. Best fit where $a=1.237, b=1.036, c_{N}=0.0015$ (the glueball contribution dominates).

\begin{tabular}{lcc}
\hline \hline Parameter & Value & Experiment \\
\hline$m_{\pi} a_{0}^{(+)}$ & -0.0029 & $0.0076 \pm 0.0031$ \\
$m_{\pi}^{3} a_{1+}^{(+)}$ & 0.047 & $0.133 \pm 0.004$ \\
$m_{\pi}^{3} a_{1-}^{(+)}$ & -0.092 & $-0.056 \pm 0.010$ \\
$m_{\pi}^{3} r_{0}^{(+)}$ & 0.012 & $-0.06 \pm 0.02$ \\
\hline \hline
\end{tabular}


Then we studied the role of $f_{0}(500)$ and $f_{0}(1710)$ in the baryonic sector of the eLSM. The nucleon and its chiral partner were incorporated into the model in the so-called mirror assignment and theoretical expressions for the pionnucleon scattering parameters, namely the isospin-even and isospin-odd scattering lengths, scattering volumes, and effective range parameters were derived. First, we presented results without the scalar four-quark and glueball state, correcting and extending results of Ref. [35]. Our results are found to be in the correct order of magnitude compared to experimental data, but there is still room for improvement.

For instance, the inclusion of the $\Delta$ resonance is expected to be important, since this state is just slightly heavier than the nucleon and it couples strongly to $N \pi$. Indeed, as a preliminary study shows [101], the effect of the $\Delta$ pushes $a_{0}^{(+)}$ toward positive values, in agreement with Refs. [96,97]. A proper study of this issue would require the inclusion of the $\Delta$ and its chiral partner into the eLSM in the framework of the mirror assignment.

Another straightforward extension of this work would be to consider the three-flavor case. Two additional resonances appear in the scalar-isoscalar sector: the strange-antistrange quarkonium [predominantly $f_{0}(1500)$ ] and the four-quark state $f_{0}(980)$ (a kaon-kaon state in the molecular picture, a $[u, s][\bar{u}, \bar{s}]+[d, s][\bar{d}, \bar{s}]$ state in the tetraquark picture). Moreover, also the quarkonium states $a_{0}(1450)$ and $K_{0}^{*}(1430)$ and the four-quark states $a_{0}(980)$ and $K_{0}^{*}(800)$ would enter this extended scenario. In this context, the difference between different internal structures of the four-quark states (mesonmeson or diquark-antidiquark) would also become visible in terms of different Clebsch-Gordon coefficients.

In the baryonic sector, an interesting future work would be to continue the nonzero-density study of Ref. [70]. Due to the recent discovery of gravitational waves emitted by neutron-star binary mergers, it is expected that the equation of state of nuclear matter at high density can be more precisely determined in the future.

\section{ACKNOWLEDGMENTS}

The authors thank the Chiral Field Theory Group, P. Kovacs, Daiki Suenaga, and S. Rechenberger, for useful discussions. F.G. acknowledges financial support from the Polish National Science Centre NCN through OPUS Project No. 2015/17/B/ST2/01625. F.G. and D.H.R. acknowledge financial support from DFG under Grant No. RI 1181/6-1. D.H.R. is partially supported by the High-end Foreign Experts project GDW20167100136 of the State Administration of Foreign Experts Affairs of China.

\section{APPENDICES}

We used Mathematica ${ }^{\circledR}$ for the numerical evaluation and the fits performed in this work. The notebooks can be found on GitHub [102].

\section{APPENDIX A: TETRAQUARK-BARYON INTERACTION TERMS}

As in the meson sector, we construct possible tetraquarkbaryon interaction terms for $N_{\mathrm{f}}=3$ and then reduce them to $N_{\mathrm{f}}=2$ in order to verify that the effective interaction of Eq. (32) can be derived within our approach. To this end, we use the formalism of Ref. [36], where four baryonic multiplets, $N_{1}, N_{2}, M_{1}$, and $M_{2}$, were constructed using the quarkdiquark picture for baryons; cf. Table III of Ref. [103] for the transformation properties under chiral symmetry, parity, and charge conjugation. We find the following terms where scalar tetraquarks couple to baryons:

$$
\begin{aligned}
\mathcal{L}_{\chi B}= & \kappa_{1} \operatorname{Tr}\left[\bar{M}_{1 \mathrm{R}} D_{\mathrm{L}} D_{\mathrm{L}}^{\dagger} N_{1 \mathrm{~L}}+\bar{M}_{2 \mathrm{~L}} D_{\mathrm{R}} D_{\mathrm{R}}^{\dagger} N_{2 \mathrm{R}}+\text { H.c. }\right] \\
& +\kappa_{2} \operatorname{Tr}\left[\bar{M}_{1 \mathrm{~L}} D_{\mathrm{R}} D_{\mathrm{R}}^{\dagger} N_{1 \mathrm{R}}+\bar{M}_{2 \mathrm{R}} D_{\mathrm{L}} D_{\mathrm{L}}^{\dagger} N_{2 \mathrm{~L}}+\text { H.c. }\right] \\
& +\left\{\kappa_{3} \operatorname{Tr}\left[\bar{M}_{1 \mathrm{R}} N_{1 \mathrm{~L}}+\bar{M}_{2 \mathrm{~L}} N_{2 \mathrm{R}}+\text { H.c. }\right]\right. \\
& \left.+\kappa_{4} \operatorname{Tr}\left[\bar{M}_{1 \mathrm{~L}} N_{1 \mathrm{R}}+\bar{M}_{2 \mathrm{R}} N_{2 \mathrm{~L}}+\text { H.c. }\right]\right\} \\
& \times \operatorname{Tr}\left[D_{\mathrm{R}} D_{\mathrm{R}}^{\dagger}+D_{\mathrm{L}} D_{\mathrm{L}}^{\dagger}\right]
\end{aligned}
$$

which reduces to Eq. (32) for two flavors using the same approach as in Sec. II B. We show this explicitly for one term:

$$
\begin{aligned}
\operatorname{Tr} & {\left[\bar{M}_{2 \mathrm{~L}} D_{\mathrm{R}} D_{\mathrm{R}}^{\dagger} N_{2 \mathrm{R}}\right] } \\
& =\operatorname{Tr}\left[\bar{M}_{2} D_{\mathrm{R}} D_{\mathrm{R}}^{\dagger} N_{2}\right]+\cdots \\
& =\frac{1}{2} \operatorname{Tr}\left[\left(\bar{B}_{M^{*}}-\bar{B}_{M}\right) D_{\mathrm{R}} D_{\mathrm{R}}^{\dagger}\left(B_{N^{*}}-B_{N}\right)\right]+\cdots \\
& =\frac{1}{4} \operatorname{Tr}\left[\left(\bar{B}_{M^{*}}-\bar{B}_{M}\right)\left(D D^{\dagger}+\cdots\right)\left(B_{N^{*}}-B_{N}\right)\right]+\cdots \\
& =\frac{1}{4} T_{a b} \operatorname{Tr}\left[\bar{B}_{M^{*}} A_{b} A_{a}^{T} B_{N}\right]+\cdots \\
& \stackrel{N_{\mathrm{f}}=2}{\longrightarrow} \frac{1}{4} \chi \bar{\psi}_{2} \psi_{1}+\cdots
\end{aligned}
$$

From the first to the second line we used the definition of the baryon fields with definite parity and charge-conjugation properties as defined in Ref. [103]. The $N_{\mathrm{f}}=2$ limit in the last line is obtained by setting $a=b=3$ and by reducing

$$
B_{M^{*}} \stackrel{N_{\mathrm{f}}=2}{\longrightarrow}\left(\begin{array}{ccc}
0 & 0 & \psi_{2,1} \\
0 & 0 & \psi_{2,2} \\
0 & 0 & 0
\end{array}\right), \quad B_{N} \stackrel{N_{\mathrm{f}}=2}{\longrightarrow}\left(\begin{array}{ccc}
0 & 0 & \psi_{1,1} \\
0 & 0 & \psi_{1,2} \\
0 & 0 & 0
\end{array}\right) .
$$

\section{APPENDIX B: MASSES AND PARAMETERS}

\section{Axial-vector-pseudoscalar mixing}

After spontaneous symmetry breaking, the field $\sigma$ is shifted by its vacuum expectation value, i.e., $\sigma \rightarrow \varphi+\sigma$. This yields mixing terms between axial-vector and pseudoscalar mesons, e.g., $\sim \vec{a}_{1}^{\mu} \cdot \partial_{\mu} \vec{\pi}$. Such terms can be eliminated by a redefinition of the fields,

$$
\vec{a}_{1}^{\mu} \rightarrow \vec{a}_{1}^{\mu}+Z w \partial^{\mu} \vec{\pi}, \quad \vec{\pi} \rightarrow Z \vec{\pi},
$$

where

$$
w=\frac{g_{1} \varphi}{m_{a_{1}}^{2}},
$$

with $m_{a_{1}}^{2}$ from Eq. (B10), and

$$
Z=\left(1-\frac{g_{1}^{2} \varphi^{2}}{m_{a_{1}}^{2}}\right)^{-1 / 2}
$$




\section{Masses}

The masses of the resonances are calculated from the second partial derivative of the potential density [which is obtained from Eqs. (8) and (25)] with respect to the corresponding fields:

$$
\begin{aligned}
& m_{\sigma}^{2}=\mu^{2}-c+3\left(\lambda_{1}+\frac{\lambda_{2}}{2}\right) \varphi^{2}-2 g_{\chi} \chi_{0}, \\
& m_{\pi}^{2}=Z^{2}\left[\mu^{2}-c+\left(\lambda_{1}+\frac{\lambda_{2}}{2}\right) \varphi^{2}-2 g_{\chi} \chi_{0}\right], \\
& m_{\eta}^{2}=Z^{2}\left[\mu^{2}+c+\left(\lambda_{1}+\frac{\lambda_{2}}{2}\right) \varphi^{2}+2 g_{\chi} \chi_{0}\right], \\
& m_{a_{0}}^{2}=\mu^{2}+c+\left(\lambda_{1}+\frac{3 \lambda_{2}}{2}\right) \varphi^{2}+2 g_{\chi} \chi_{0}, \\
& m_{\rho}^{2}=m_{1}^{2}+\left(h+h_{3}\right) \frac{\varphi^{2}}{2}+2 g_{\mathrm{AV}} \chi_{0}, \\
& m_{\omega}^{2}=m_{1}^{2}+\left(h+h_{3}\right) \frac{\varphi^{2}}{2}-2 g_{\mathrm{AV}} \chi_{0} \\
& m_{a_{1}}^{2}=m_{1}^{2}+\left(2 g_{1}^{2}+h-h_{3}\right) \frac{\varphi^{2}}{2}+2 g_{\mathrm{AV} \chi_{0}}, \\
& m_{f_{1}}^{2}=m_{1}^{2}+\left(2 g_{1}^{2}+h-h_{3}\right) \frac{\varphi^{2}}{2}-2 g_{\mathrm{AV} \chi_{0} .}
\end{aligned}
$$

\section{Parameters}

The vacuum expectation value of $\sigma, \varphi=Z f_{\pi}$, is determined from the axial current. The vacuum expectation value of the scalar four-quark state is determined by the minimum of the potential density:

$$
\chi_{0}\left(g_{\chi}, m_{\chi}^{2}\right)=\frac{g_{\chi} \varphi^{2}}{m_{\chi}^{2}}
$$

We can use the masses, whose values are given by experimental data, to fix some of the model parameters:

$$
\begin{aligned}
\lambda_{2}= & \frac{1}{\varphi^{2}}\left(m_{a_{0}}^{2}-\frac{m_{\eta}^{2}}{Z^{2}}\right) \\
h_{3}= & \frac{1}{\varphi^{2}}\left(m_{\rho}^{2}-m_{a_{1}}^{2}+g_{1}^{2} \varphi^{2}\right) \\
c= & c\left(g_{\chi}, m_{\chi}^{2}\right)=\frac{1}{2 Z^{2}}\left(m_{\eta}^{2}-m_{\pi}^{2}\right)-2 g_{\chi} \chi_{0}\left(g_{\chi}, m_{\chi}^{2}\right) \\
\mu^{2}= & \mu^{2}\left(\lambda_{1}\right)=\frac{1}{2}\left[\frac{1}{Z^{2}}\left(m_{\eta}^{2}+m_{\pi}^{2}\right)-\varphi^{2}\left(2 \lambda_{1}+\lambda_{2}\right)\right] \\
m_{1}^{2}= & m_{1}^{2}\left(g_{\chi}, m_{\chi}^{2}, h\right) \\
= & \frac{1}{2}\left[m_{\rho}^{2}+m_{a_{1}}^{2}-4 g_{\mathrm{AV}}\left(g_{\chi}, m_{\chi}^{2}\right) \chi_{0}\left(g_{\chi}, m_{\chi}^{2}\right)\right. \\
& \left.-\varphi^{2}\left(g_{1}^{2}+h\right)\right], \\
g_{\mathrm{AV}}= & g_{\mathrm{AV}}\left(g_{\chi}, m_{\chi}^{2}\right)=\frac{m_{\rho}^{2}-m_{\omega}^{2}}{4 \chi_{0}\left(g_{\chi}, m_{\chi}^{2}\right)}=m_{\chi}^{2} \frac{m_{\rho}^{2}-m_{\omega}^{2}}{4 g_{\chi} \varphi^{2}}
\end{aligned}
$$

where we used Eq. (B12) in the last step of the last line. Note that $g_{\mathrm{AV}}$ and $g_{\chi}$ are inversely proportional to each other, i.e., a small value of $g_{\chi}$ requires a large value of $g_{\mathrm{AV}}$ and vice versa.

\section{APPENDIX C: MESON SECTOR WITHOUT DYNAMICAL SCALAR GLUEBALL}

\section{Scalar mixing angle}

The shift $\sigma \rightarrow \varphi+\sigma$ and $\chi \rightarrow \chi_{0}+\chi$ leads to a nondiagonal mass matrix. We rotate the fields via an $\operatorname{SO}(2)$ transformation,

$$
\left(\begin{array}{l}
\chi \\
\sigma
\end{array}\right)=\left(\begin{array}{cc}
\cos \theta & -\sin \theta \\
\sin \theta & \cos \theta
\end{array}\right)\left(\begin{array}{c}
H \\
S
\end{array}\right),
$$

and demand that the mass matrix in the basis of the new fields, $H$ and $S$, must be diagonal. This leads to a mixing angle

$$
\theta=\frac{1}{2} \arctan \frac{4 g_{\chi} \varphi}{m_{\sigma}^{2}-m_{\chi}^{2}} .
$$

We then relate the physical masses to the unphysical ones:

$$
\begin{aligned}
& M_{H}^{2}=m_{\chi}^{2} \cos ^{2} \theta+m_{\sigma}^{2} \sin ^{2} \theta-2 g_{\chi} \varphi \sin 2 \theta, \\
& M_{S}^{2}=m_{\sigma}^{2} \cos ^{2} \theta+m_{\chi}^{2} \sin ^{2} \theta+2 g_{\chi} \varphi \sin 2 \theta .
\end{aligned}
$$

\section{Decay widths}

We are interested in the decay of the scalars $\sigma$ and $\chi$ into two pions. The information of this decay is contained in the Lagrangians

$$
\begin{aligned}
& \mathcal{L}_{\sigma \rightarrow \pi \pi}=A_{\sigma} \sigma \vec{\pi}^{2}+B_{\sigma} \sigma \partial_{\mu} \vec{\pi} \cdot \partial^{\mu} \vec{\pi}-C_{\sigma} \sigma \vec{\pi} \cdot \square \vec{\pi}, \\
& \mathcal{L}_{\chi \rightarrow \pi \pi}=A_{\chi} \chi \vec{\pi}^{2}+B_{\chi} \chi \partial_{\mu} \vec{\pi} \cdot \partial^{\mu} \vec{\pi},
\end{aligned}
$$

where

$$
\begin{aligned}
& A_{\sigma}=-Z^{2} \varphi\left(\lambda_{1}+\frac{\lambda_{2}}{2}\right), \\
& B_{\sigma}=Z^{2} w\left[-2 g_{1}+\frac{w \varphi}{2}\left(2 g_{1}^{2}+h-h_{3}\right)\right], \\
& C_{\sigma}=-g_{1} Z^{2} w \\
& A_{\chi}=g_{\chi} Z^{2} \\
& B_{\chi}=g_{\mathrm{AV}} w^{2} Z^{2} .
\end{aligned}
$$

The Feynman amplitudes of the physical fields are obtained from the mixings

$$
\begin{aligned}
\mathcal{M}_{H \pi \pi} & =\mathcal{M}_{\sigma \pi \pi}\left(m_{H}\right) \sin \theta+\mathcal{M}_{\chi \pi \pi}\left(m_{H}\right) \cos \theta, \\
\mathcal{M}_{S \pi \pi} & =\mathcal{M}_{\sigma \pi \pi}\left(m_{S}\right) \cos \theta-\mathcal{M}_{\chi \pi \pi}\left(m_{S}\right) \sin \theta .
\end{aligned}
$$

where

$$
\begin{gathered}
-i \mathcal{M}_{\sigma \pi \pi}\left(m_{X}\right)=i\left(A_{\sigma}-B_{\sigma} \frac{m_{X}^{2}-2 m_{\pi}^{2}}{2}-C_{\sigma} m_{\pi}^{2}\right), \\
-i \mathcal{M}_{\chi \pi \pi}\left(m_{X}\right)=i\left(A_{\chi}-B_{\chi} \frac{m_{X}^{2}-2 m_{\pi}^{2}}{2}\right) .
\end{gathered}
$$

The decay widths are given by

$$
\Gamma_{H \rightarrow \pi \pi}=3 \frac{k_{f}\left(m_{H}, m_{\pi}, m_{\pi}\right)}{4 \pi m_{H}^{2}}\left|-i \mathcal{M}_{H \pi \pi}\right|^{2},
$$



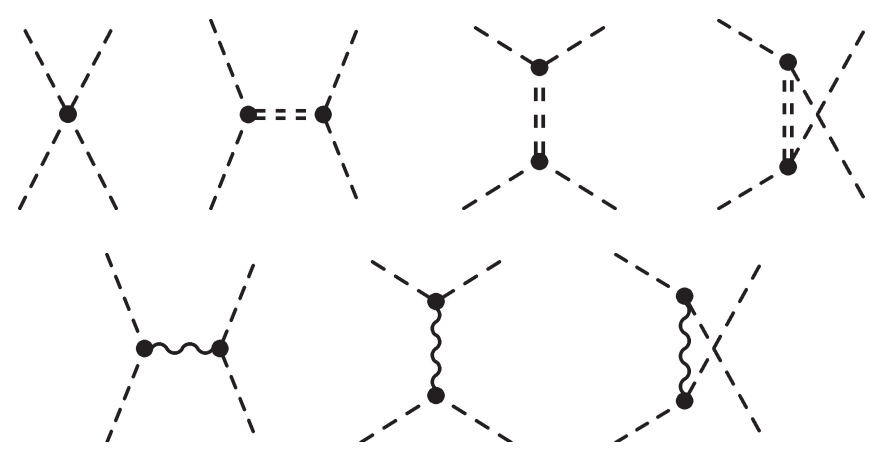

FIG. 1. The dashed lines correspond to the pion, the wavy line to the $\rho$ meson, and the double-dashed line to the $\sigma$ or the $\chi$. (Each diagram with internal double-dashed lines occurs twice, once with the exchange of $\sigma$ and once with the exchange of $\chi$.)

$$
\Gamma_{S \rightarrow \pi \pi}=3 \frac{k_{f}\left(m_{S}, m_{\pi}, m_{\pi}\right)}{4 \pi m_{S}^{2}}\left|-i \mathcal{M}_{S \pi \pi}\right|^{2} .
$$

\section{Pion-pion scattering}

The scattering amplitude is calculated from the tree-level amplitudes in Fig. 1. The pion-pion interaction consists of three parts,

$$
\mathcal{L}_{\pi \pi}=\mathcal{L}_{4 \pi}+\mathcal{L}_{\sigma \pi \pi}+\mathcal{L}_{\rho \pi \pi},
$$

which can be extracted from the eLSM Lagrangian (8). From this we obtain the scattering amplitude

$$
\begin{aligned}
\mathcal{M}_{\pi \pi}(s, t, u)= & i \delta_{a b} \delta_{c d} A(s, t, u)+i \delta_{a c} \delta_{b d} A(t, u, s) \\
& +i \delta_{a d} \delta_{b c} A(u, s, t),
\end{aligned}
$$

where

$$
\begin{aligned}
A(s, t, u)= & \left(g_{1}^{2}-h_{3}\right) Z^{4} w^{2} s-2\left(\lambda_{1}+\frac{\lambda_{2}}{2}\right) Z^{4} \\
& -\left(h+h_{3}\right) Z^{4} w^{2}\left(s-2 m_{\pi}^{2}\right) \\
& -\frac{1}{s-m_{\sigma}^{2}}\left[-2 m_{\pi}^{2} C_{\sigma}+B_{\sigma}\left(2 m_{\pi}^{2}-s\right)+2 A_{\sigma}\right]^{2} \\
& +\left(A_{\rho}+B_{\rho} \frac{t}{2}\right)^{2} \frac{u-s}{t-m_{\rho}^{2}} \\
& +\left(A_{\rho}+B_{\rho} \frac{u}{2}\right)^{2} \frac{t-s}{u-m_{\rho}^{2}}
\end{aligned}
$$

The coefficients $A_{\sigma}, B_{\sigma}$, and $C_{\sigma}$ are given in Eqs. (C7)-(C9), while $A_{\rho}=g_{1} Z^{2} m_{\rho}^{2} / m_{a_{1}}^{2}$ and $B_{\rho}=g_{2} Z^{2} w^{2}$.

The scattering amplitude in the $I=0$ channel is given by the relation [104]

$$
T^{0}(s, t, u)=3 A(s, t, u)+A(t, u, s)+A(u, s, t),
$$

from which the isospin-zero scattering length is computed as

$$
m_{\pi} a_{0}^{0}=\frac{1}{32 \pi} T^{0}\left(4 m_{\pi}^{2}, 0,0\right) .
$$

On the other hand, the scattering amplitude for isospin $I=$ 2 is given by

$$
T^{2}(s, t, u)=2 A(u, s, t),
$$

and the $s$-wave scattering length is extracted as

$$
m_{\pi} a_{0}^{2}=\frac{1}{32 \pi} T^{2}\left(4 m_{\pi}^{2}, 0,0\right) .
$$

After the introduction of the scalar four-quark field, the term

$$
\frac{1}{s-m_{\sigma}^{2}}\left[-2 m_{\pi}^{2} C_{\sigma}+B_{\sigma}\left(2 m_{\pi}^{2}-s\right)+2 A_{\sigma}\right]^{2}
$$

in Eq. (C20) is replaced by

$$
\begin{aligned}
& \frac{1}{s-M_{H}^{2}}\left[-2 m_{\pi}^{2} C_{H}+B_{H}\left(2 m_{\pi}^{2}-s\right)+2 A_{H}\right]^{2} \\
& \quad+\frac{1}{s-M_{S}^{2}}\left[-2 m_{\pi}^{2} C_{S}+B_{S}\left(2 m_{\pi}^{2}-s\right)+2 A_{S}\right]^{2} .
\end{aligned}
$$

The new coefficients are given as

$$
\begin{aligned}
A_{H} & =A_{\sigma} \sin \theta+A_{\chi} \cos \theta, \\
A_{S} & =A_{\sigma} \cos \theta-A_{\chi} \sin \theta, \\
B_{H} & =B_{\sigma} \sin \theta+B_{\chi} \cos \theta, \\
B_{S} & =B_{\sigma} \cos \theta-B_{\chi} \sin \theta, \\
C_{H} & =C_{\sigma} \sin \theta, \\
C_{S} & =C_{\sigma} \cos \theta .
\end{aligned}
$$

Thus, at threshold ( $s \equiv 4 m_{\pi}^{2}, t=0, u=0$ ), we obtain the scattering lengths

$$
\begin{aligned}
m_{\pi} a_{0}^{0}= & \frac{1}{32 \pi}\left\{12\left(g_{1}^{2}-h_{3}\right) Z^{4} w^{2} m_{\pi}^{2}-10\left(\lambda_{1}+\frac{\lambda_{2}}{2}\right) Z^{4}-2\left(h+h_{3}\right) Z^{4} w^{2} m_{\pi}^{2}+\frac{12}{M_{H}^{2}-4 m_{\pi}^{2}}\left[\left(B_{H}+C_{H}\right) m_{\pi}^{2}-A_{H}\right]^{2}\right. \\
& \left.+\frac{12}{M_{S}^{2}-4 m_{\pi}^{2}}\left[\left(B_{S}+C_{S}\right) m_{\pi}^{2}-A_{S}\right]^{2}+\frac{8}{M_{H}^{2}}\left[\left(B_{H}-C_{H}\right) m_{\pi}^{2}+A_{H}\right]^{2}+\frac{8}{M_{S}^{2}}\left[\left(B_{S}-C_{S}\right) m_{\pi}^{2}+A_{S}\right]^{2}+16 g_{1}^{2} Z^{4} m_{\pi}^{2} \frac{m_{\rho}^{2}}{m_{a_{1}}^{4}}\right\}
\end{aligned}
$$

and

$$
\begin{aligned}
m_{\pi} a_{0}^{2}= & \frac{1}{16 \pi}\left\{-2\left(\lambda_{1}+\frac{\lambda_{2}}{2}\right) Z^{4}+2\left(h_{1}+h_{2}+h_{3}\right) Z^{4} w^{2} m_{\pi}^{2}-4 g_{1}^{2} Z^{4} m_{\pi}^{2} \frac{m_{\rho}^{2}}{m_{a_{1}}^{4}}\right. \\
& \left.+\frac{4}{M_{H}^{2}}\left[\left(B_{H}-C_{H}\right) m_{\pi}^{2}+A_{H}\right]^{2}+\frac{4}{M_{S}^{2}}\left[\left(B_{S}-C_{S}\right) m_{\pi}^{2}+A_{S}\right]^{2}\right\} .
\end{aligned}
$$




\section{APPENDIX D: MESON SECTOR WITH DYNAMICAL \\ SCALAR GLUEBALL}

\section{Decay widths}

From Eqs. (8) and (25) we read off the relevant terms for the decay $\Gamma_{G \rightarrow \pi \pi}$ :

$$
\begin{aligned}
\mathcal{L}_{\text {Glueball-Int }}= & -\frac{Z^{2}}{G_{0}}\left(\mu^{2}-g_{\chi} \chi_{0}\right) G \vec{\pi}^{2} \\
& +\frac{Z^{2} w^{2}}{G_{0}}\left(m_{1}^{2}+g_{\mathrm{AV}} \chi_{0}\right) G\left(\partial_{\mu} \pi\right)^{2} .
\end{aligned}
$$

This yields another contribution which is of the same form as the $\sigma \vec{\pi}^{2}$ and the $\chi \vec{\pi}^{2}$ interaction. The corresponding partial amplitudes are

$$
\begin{aligned}
& A_{G}=-\frac{Z^{2}}{G_{0}}\left(\mu^{2}-g_{\chi} \chi_{0}\right), \\
& B_{G}=\frac{Z^{2} w^{2}}{G_{0}}\left(m_{1}^{2}+g_{\mathrm{AV}} \chi_{0}\right) .
\end{aligned}
$$

We define the inverse mixing matrix as $Q=O^{T}$ [see Eq. (30)] such that the new coefficients are given as

$$
\begin{aligned}
A_{H} & =Q_{11} A_{\chi}+Q_{21} A_{\sigma}+Q_{31} A_{G}, \\
A_{S} & =Q_{12} A_{\chi}+Q_{22} A_{\sigma}+Q_{32} A_{G}, \\
A_{G^{\prime}} & =Q_{13} A_{\chi}+Q_{23} A_{\sigma}+Q_{33} A_{G}, \\
B_{H} & =Q_{11} B_{\chi}+Q_{21} B_{\sigma}+Q_{31} B_{G}, \\
B_{S} & =Q_{12} B_{\chi}+Q_{22} B_{\sigma}+Q_{32} B_{G},
\end{aligned}
$$

$$
\begin{aligned}
B_{G^{\prime}} & =Q_{13} B_{\chi}+Q_{23} B_{\sigma}+Q_{33} B_{G}, \\
C_{H} & =Q_{21} C_{\sigma}, \\
C_{S} & =Q_{22} C_{\sigma}, \\
C_{G^{\prime}} & =Q_{23} C_{\sigma} .
\end{aligned}
$$

With these amplitudes the decay widths are given by

$$
\begin{aligned}
\Gamma_{H \rightarrow \pi \pi} & =3 \frac{k_{f}\left(M_{H}, m_{\pi}, m_{\pi}\right)}{4 \pi M_{H}^{2}}\left|-i \mathcal{M}_{H}\right|^{2}, \\
\Gamma_{S \rightarrow \pi \pi} & =3 \frac{k_{f}\left(M_{S}, m_{\pi}, m_{\pi}\right)}{4 \pi M_{S}^{2}}\left|-i \mathcal{M}_{S}\right|^{2}, \\
\Gamma_{G^{\prime} \rightarrow \pi \pi} & =3 \frac{k_{f}\left(M_{G}^{\prime}, m_{\pi}, m_{\pi}\right)}{4 \pi M_{G^{\prime}}^{2}}\left|-i \mathcal{M}_{G^{\prime}}\right|^{2},
\end{aligned}
$$

where

$$
\begin{aligned}
-i \mathcal{M}_{H} & =i\left(A_{H}-B_{H} \frac{M_{H}^{2}-2 m_{\pi}^{2}}{2}-C_{H} m_{\pi}^{2}\right), \\
-i \mathcal{M}_{S} & =i\left(A_{S}-B_{S} \frac{M_{S}^{2}-2 m_{\pi}^{2}}{2}-C_{S} m_{\pi}^{2}\right), \\
-i \mathcal{M}_{G^{\prime}} & =i\left(A_{G^{\prime}}-B_{G^{\prime}} \frac{M_{G^{\prime}}^{2}-2 m_{\pi}^{2}}{2}-C_{G^{\prime}} m_{\pi}^{2}\right) .
\end{aligned}
$$

\section{Pion-pion scattering}

For the pion-pion scattering amplitude, we simply need to add the corresponding expression for the scalar glueball exchange to Eq. (C26). Then, the dimensionless scattering lengths are given as

$$
\begin{aligned}
m_{\pi} a_{0}^{0}= & \frac{1}{32 \pi}\left\{12\left(g_{1}^{2}-h_{3}\right) Z^{4} w^{2} m_{\pi}^{2}-10\left(\lambda_{1}+\frac{\lambda_{2}}{2}\right) Z^{4}-2\left(h+h_{3}\right) Z^{4} w^{2} m_{\pi}^{2}+\frac{12}{M_{H}^{2}-4 m_{\pi}^{2}}\left[\left(B_{H}+C_{H}\right) m_{\pi}^{2}-A_{H}\right]^{2}\right. \\
& +\frac{12}{M_{S}^{2}-4 m_{\pi}^{2}}\left[\left(B_{S}+C_{S}\right) m_{\pi}^{2}-A_{S}\right]^{2}+\frac{12}{M_{G^{\prime}}^{2}-4 m_{\pi}^{2}}\left[\left(B_{G^{\prime}}+C_{G^{\prime}}\right) m_{\pi}^{2}-A_{G^{\prime}}\right]^{2}+\frac{8}{M_{H}^{2}}\left[\left(B_{H}-C_{H}\right) m_{\pi}^{2}+A_{H}\right]^{2} \\
& \left.+\frac{8}{M_{S}^{2}}\left[\left(B_{S}-C_{S}\right) m_{\pi}^{2}+A_{S}\right]^{2}+\frac{8}{M_{G^{\prime}}^{2}}\left[\left(B_{G^{\prime}}-C_{G^{\prime}}\right) m_{\pi}^{2}+A_{G^{\prime}}\right]^{2}+16 g_{1}^{2} Z^{4} m_{\pi}^{2} \frac{m_{\rho}^{2}}{m_{a_{1}}^{4}}\right\}
\end{aligned}
$$

and

$$
\begin{aligned}
m_{\pi} a_{0}^{2}= & \frac{1}{16 \pi}\left\{-2\left(\lambda_{1}+\frac{\lambda_{2}}{2}\right) Z^{4}+2\left(h+h_{3}\right) Z^{4} w^{2} m_{\pi}^{2}-4 g_{1}^{2} Z^{4} m_{\pi}^{2} \frac{m_{\rho}^{2}}{m_{a_{1}}^{4}}\right. \\
& \left.+\frac{4}{M_{H}^{2}}\left[\left(B_{H}-C_{H}\right) m_{\pi}^{2}+A_{H}\right]^{2}+\frac{4}{M_{S}^{2}}\left[\left(B_{S}-C_{S}\right) m_{\pi}^{2}+A_{S}\right]^{2}+\frac{4}{M_{G^{\prime}}^{2}}\left[\left(B_{G^{\prime}}-C_{G^{\prime}}\right) m_{\pi}^{2}+A_{G^{\prime}}\right]^{2}\right\} .
\end{aligned}
$$

\section{APPENDIX E: PION-NUCLEON SCATTERING PARAMETERS}

The Feynman diagrams which contribute to the pionnucleon scattering amplitude are shown in Fig. 2. The isospineven and isospin-odd scattering lengths, scattering volumes, and effective range parameters can be calculated using [105]

$$
\begin{aligned}
& a_{0}^{( \pm)}=\eta\left(A_{0}^{( \pm)}+m_{\pi} B_{0}^{( \pm)}\right), \\
& a_{1+}^{( \pm)}=\frac{2}{3} \eta C_{0}^{( \pm)},
\end{aligned}
$$

$$
\begin{aligned}
a_{1-}^{( \pm)}= & \frac{2}{3} \eta C_{0}^{( \pm)}-\frac{\eta}{4 m_{N}^{2}}\left[A_{0}^{( \pm)}-\left(2 m_{N}+m_{\pi}\right) B_{0}^{( \pm)}\right], \\
r_{0}^{( \pm)}= & \eta\left\{-2 C_{0}^{( \pm)}+\frac{\left(m_{N}+m_{\pi}\right)^{2}}{m_{N} m_{\pi}} D_{0}^{( \pm)}-\frac{1}{2 m_{N} m_{\pi}}\right. \\
& \left.\times\left[\left(1-\frac{m_{\pi}}{2 m_{N}}\right) A_{0}^{( \pm)}-\left(m_{N}+\frac{m_{\pi}^{2}}{2 m_{N}}\right) B_{0}^{( \pm)}\right]\right\},
\end{aligned}
$$



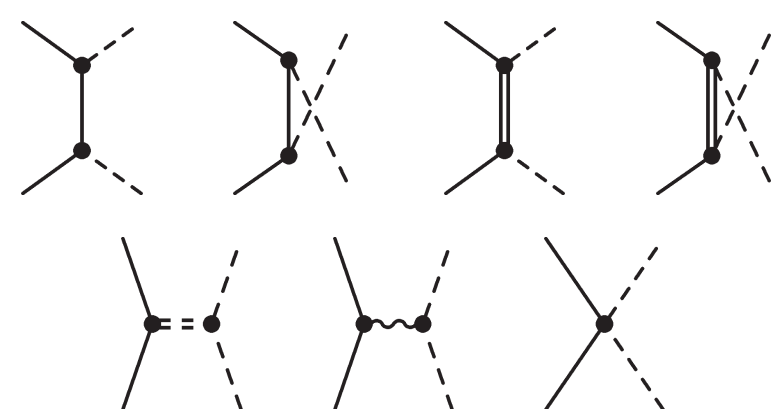

FIG. 2. Pion-nucleon scattering diagrams at tree level. The solid line represents the nucleon, the double line its chiral partner, the dashed line the pion, the double-dashed the scalar-isoscalars $H, S$, and $G^{\prime}$, and the wavy line the $\rho$ meson.

where

$$
\eta=\frac{1}{4 \pi\left(1+\frac{m_{\pi}}{m_{N}}\right)},
$$

$$
\begin{aligned}
& C_{0}^{( \pm)}=\left.\frac{\partial}{\partial t}\left(A^{( \pm)}+m_{\pi} B^{ \pm}\right)\right|_{t=0}, \\
& D_{0}^{( \pm)}=\left.\frac{\partial}{\partial s}\left(A^{( \pm)}+m_{\pi} B^{ \pm}\right)\right|_{t=0}
\end{aligned}
$$

The partial amplitudes $A^{( \pm)}$and $B^{( \pm)}$can be extracted by rewriting the scattering amplitude into the form

$$
\begin{aligned}
T_{a b}= & {\left[A^{(+)}+\frac{\left(q_{1}+q_{2}\right)}{2} B^{(+)}\right] \delta_{a b} } \\
& +\left[A^{(-)}+\frac{\left(q_{1}+q_{2}\right)}{2} B^{(-)}\right] i \epsilon_{b a c} \tau_{c}
\end{aligned}
$$

where $a$ and $b$ are the isospin indices of the initial and final pion states.

The correct result for the partial amplitudes for the model of Ref. [35] reads

$$
\begin{aligned}
A^{(+)}= & 4\left(g_{\pi N} g_{\partial \pi N}+g_{\partial \pi N}^{2} m_{N}-g_{N^{*} \pi} g_{N^{*} \partial \pi}\right)-2 g_{N^{*} \partial \pi}^{2}\left(m_{N^{*}}-m_{N}\right)+2 g_{N N \pi \pi} \\
& +\left\{-g_{N^{*} \pi}^{2}\left(m_{N}+m_{N^{*}}\right)+\left(m_{N}^{2}-m_{N^{*}}^{2}\right)\left[2 g_{N^{*} \pi} g_{N^{*} \partial \pi}+g_{N^{*} \partial \pi}^{2}\left(m_{N^{*}}-m_{N}\right)\right]\right\}\left(\frac{1}{s-m_{N^{*}}^{2}}+\frac{1}{u-m_{N^{*}}^{2}}\right) \\
& -\frac{2 g_{N \sigma}}{t-m_{\sigma}^{2}}\left[g_{\pi \sigma}+g_{\partial \pi \sigma}\left(m_{\pi}^{2}-\frac{t}{2}\right)+g_{\partial \sigma \pi} \frac{t}{2}\right] \\
A^{(-)}= & \left\{-g_{N^{*} \pi}^{2}\left(m_{N}+m_{N^{*}}\right)+\left(m_{N}^{2}-m_{N^{*}}^{2}\right)\left[2 g_{N^{*} \pi} g_{N^{*} \partial \pi}+g_{N^{*} \partial \pi}^{2}\left(m_{N^{*}}-m_{N}\right)\right]\right\}\left(\frac{1}{s-m_{N^{*}}^{2}}-\frac{1}{u-m_{N^{*}}^{2}}\right), \\
B^{(+)}= & -\left(g_{\pi N}+2 g_{\partial \pi N} m_{N}\right)^{2}\left(\frac{1}{s-m_{N}^{2}}-\frac{1}{u-m_{N}^{2}}\right)-\left[g_{N^{*} \pi}-g_{N^{*} \partial \pi}\left(m_{N^{*}}-m_{N}\right)\right]^{2}\left(\frac{1}{s-m_{N^{*}}^{2}}-\frac{1}{u-m_{N^{*}}^{2}}\right), \\
B^{(-)}= & -\left(g_{\pi N}+2 g_{\partial \pi N} m_{N}\right)^{2}\left(\frac{1}{s-m_{N}^{2}}+\frac{1}{u-m_{N}^{2}}\right)-\left[g_{N^{*} \pi}+g_{N^{*} \partial \pi}\left(m_{N^{*}}-m_{N}\right)\right]^{2}\left(\frac{1}{s-m_{N^{*}}^{2}}+\frac{1}{u-m_{N^{*}}^{2}}\right) \\
& -2 g_{\partial \pi N}^{2}-2 g_{N^{*} \partial \pi}^{2}+\frac{2 g_{N \rho}}{t-m_{\rho}^{2}}\left(g_{\pi \rho}+g_{\partial \pi \partial \rho} \frac{t}{2}\right) .
\end{aligned}
$$

The couplings can be read off from the meson and the baryon Lagrangian:

$$
\begin{aligned}
g_{\pi \rho} & =-g_{1} Z^{2}+g_{1}^{2} \varphi w Z^{2}-\varphi w Z^{2}\left[m_{\rho}^{2}-m_{a_{1}}^{2}+\left(g_{1} \varphi\right)^{2}\right] \frac{1}{\varphi^{2}}=-g_{1} Z^{2} \frac{m_{\rho}^{2}}{m_{a_{1}}^{2}} \\
g_{\partial \pi \sigma} & =g_{1} w Z^{2}\left(g_{1} \varphi w-1\right)+\frac{\varphi}{2} w^{2} Z^{2}\left(h_{1}+h_{2}-h_{3}\right), \\
g_{\pi \sigma} & =-\varphi Z^{2}\left(\lambda_{1}+\frac{\lambda_{2}}{2}\right)=-\frac{Z}{2 f_{\pi}}\left(m_{\sigma}^{2}-\frac{m_{\pi}^{2}}{Z^{2}}\right), \\
g_{\partial \sigma \pi} & =g_{1} w Z^{2} \\
g_{\partial \pi \partial \rho} & =g_{2} Z^{2} w^{2} \\
g_{\chi \pi} & =g_{\chi} Z^{2}, \\
g_{\chi \partial \pi} & =g_{\mathrm{AV}} Z^{2} w^{2}, \\
g_{\pi N} & =-Z \frac{e^{\delta} \hat{g}_{1}+e^{-\delta} \hat{g}_{2}}{4 \cosh \delta}, \\
g_{\pi N N^{*}} & =-g_{\pi N^{*} N}=Z \frac{-\hat{g}_{1}+\hat{g}_{2}}{4 \cosh \delta}
\end{aligned}
$$




$$
\begin{aligned}
g_{\partial \pi N} & =Z w \frac{e^{\delta} c_{1}-e^{-\delta} c_{2}}{4 \cosh \delta}, \\
g_{\partial \pi N N^{*}} & =g_{\partial \pi N^{*} N}=-Z w \frac{c_{1}+c_{2}}{4 \cosh \delta} .
\end{aligned}
$$

From this we obtain the isospin-even and isospin-odd scattering parameters:

$$
\begin{aligned}
a_{0}^{(+)}= & \frac{1}{4 \pi\left(1+\frac{m_{\pi}}{m_{N}}\right)}\left(\frac{Z}{2 \cosh \delta}\right)^{2}\left(-\frac{1}{2}\left(\hat{g}_{1}-\hat{g}_{2}\right)^{2}\left[1-\frac{Z f_{\pi}}{2} w\left(c_{1}+c_{2}\right)\right]^{2} \frac{\left(m_{N^{*}}+m_{N}\right)\left(m_{N}^{2}+m_{\pi}^{2}-m_{N^{*}}^{2}\right)}{\left(m_{N}^{2}+m_{\pi}^{2}-m_{N^{*}}^{2}\right)^{2}-4 m_{N}^{2} m_{\pi}^{2}}\right. \\
& -w\left(c_{1}+c_{2}\right)\left(\hat{g}_{1}-\hat{g}_{2}\right)\left[1-\frac{Z f_{\pi}}{4} w\left(c_{1}+c_{2}\right)\right]-w\left(c_{1} e^{\delta}-c_{2} e^{-\delta}\right)\left[\hat{g}_{1} e^{\delta}+\hat{g}_{2} e^{-\delta}-w m_{N}\left(c_{1} e^{\delta}-c_{2} e^{-\delta}\right)\right] \\
& +\left(\hat{g}_{1} e^{\delta}-\hat{g}_{2} e^{-\delta}\right) \frac{\cosh \delta}{Z f_{\pi}}\left\{1+\frac{m_{\pi}^{2}}{m_{\sigma}^{2} Z^{4}}\left[Z^{2}-2-2\left(Z^{2}-1\right)\left(1-\frac{Z^{2} m_{1}^{2}}{m_{a_{1}}^{2}}\right)\right]\right\}+m_{\pi}\left\{\left(\hat{g}_{1}-\hat{g}_{2}\right)^{2}\left[1-\frac{Z f_{\pi}}{2} w\left(c_{1}+c_{2}\right)\right]^{2}\right. \\
& \left.\left.\times \frac{m_{N} m_{\pi}}{\left(m_{N}^{2}+m_{\pi}^{2}-m_{N^{*}}^{2}\right)^{2}-4 m_{N}^{2} m_{\pi}^{2}}+\left[\hat{g}_{1} e^{\delta}+\hat{g}_{2} e^{-\delta}-2 w m_{N}\left(c_{1} e^{\delta}-c_{2} e^{-\delta}\right)\right]^{2} \frac{m_{N}}{m_{\pi}} \frac{1}{m_{\pi}^{2}-4 m_{N}^{2}}\right\}\right) .
\end{aligned}
$$

Notice the change of sign in front of the third term in brackets in the third line as compared to Eq. (18) of Ref. [35]. The correct result for the isospin-odd scattering length reads

$$
\begin{aligned}
a_{0}^{(-)}= & \frac{1}{4 \pi\left(1+\frac{m_{\pi}}{m_{N}}\right)}\left(\frac{Z}{2 \cosh \delta}\right)^{2}\left(\left(\hat{g}_{1}-\hat{g}_{2}\right)^{2}\left[1-\frac{Z f_{\pi}}{2} w\left(c_{1}+c_{2}\right)\right]^{2} \frac{\left(m_{N}+m_{N^{*}}\right) m_{N} m_{\pi}}{\left(m_{N}^{2}+m_{\pi}^{2}-m_{N^{*}}^{2}\right)^{2}-4 m_{N}^{2} m_{\pi}^{2}}\right. \\
& +\frac{m_{\pi}}{2}\left\{-\left(\hat{g}_{1}-\hat{g}_{2}\right)^{2}\left[1-\frac{Z f_{\pi}}{2} w\left(c_{1}+c_{2}\right)\right]^{2} \frac{m_{N}^{2}+m_{\pi}^{2}-m_{N^{*}}^{2}}{\left(m_{N}^{2}+m_{\pi}^{2}-m_{N^{*}}^{2}\right)^{2}-4 m_{N}^{2} m_{\pi}^{2}}-\left[\hat{g}_{1} e^{\delta}+\hat{g}_{2} e^{-\delta}-2 w m_{N}\left(c_{1} e^{\delta}-c_{2} e^{-\delta}\right)\right]^{2}\right. \\
& \left.\left.\frac{1}{m_{\pi}^{2}-4 m_{N}^{2}}-w^{2}\left[\left(c_{1}+c_{2}\right)^{2}+\left(c_{1} e^{\delta}-c_{2} e^{-\delta}\right)^{2}\right]+4 \cosh \delta \frac{g_{1}}{m_{a_{1}}^{2}}\left(c_{1} e^{\delta}+c_{2} e^{-\delta}\right)\right\}\right) .
\end{aligned}
$$

Errors in Eq. (19) of Ref. [35] were (i) the sign of the first term in braces, (ii) the sign in front of the second term in the second set of brackets in the third line, (iii) the coefficient of the last term $\left(1 / m_{a_{1}}^{2}\right.$ instead of $\left.1 / Z^{2} m_{\rho}^{2}\right)$, and (iv) the sign in front of the last term in parentheses in the fourth line.

In order to obtain the expressions for the scattering parameters for our model with scalar four-quark state and dynamical scalar glueball we need to modify the pion-nucleon scattering amplitudes by replacing the last term of $A^{(+)}$with the expression

$$
\begin{aligned}
& -\frac{2 g_{N H}}{t-M_{H}^{2}}\left[g_{\pi H}+g_{\partial л H}\left(m_{\pi}^{2}-\frac{t}{2}\right)+g_{\partial H \pi} \frac{t}{2}\right] \\
& -\frac{2 g_{N S}}{t-M_{S}^{2}}\left[g_{\pi S}+g_{\partial \pi S}\left(m_{\pi}^{2}-\frac{t}{2}\right)+g_{\partial S \pi} \frac{t}{2}\right] \\
& -\frac{2 g_{N G^{\prime}}}{t-M_{G^{\prime}}^{2}}\left[g_{\pi G^{\prime}}+g_{\partial \pi G^{\prime}}\left(m_{\pi}^{2}-\frac{t}{2}\right)+g_{\partial G^{\prime} \pi} \frac{t}{2}\right],
\end{aligned}
$$

where

$$
\begin{aligned}
& g_{N H}=Q_{11} g_{N \chi}+Q_{21} g_{N \sigma}+Q_{31} g_{N G}, \\
& g_{N S}=Q_{12} g_{N \chi}+Q_{22} g_{N \sigma}+Q_{32} g_{N G}, \\
& g_{N G^{\prime}}=Q_{13} g_{N \chi}+Q_{23} g_{N \sigma}+Q_{33} g_{N G}, \\
& g_{\pi H}=Q_{11} g_{\pi \chi}+Q_{21} g_{\pi \sigma}+Q_{31} g_{\pi G},
\end{aligned}
$$

$$
\begin{aligned}
g_{\pi S} & =Q_{12} g_{\pi \chi}+Q_{22} g_{\pi \sigma}+Q_{32} g_{\pi G}, \\
g_{\pi G^{\prime}} & =Q_{13} g_{\pi \chi}+Q_{23} g_{\pi \sigma}+Q_{33} g_{\pi G}, \\
g_{\partial \pi H} & =Q_{11} g_{\partial \pi \chi}+Q_{21} g_{\partial \pi \sigma}+Q_{31} g_{\partial \pi G}, \\
g_{\partial \pi S} & =Q_{12} g_{\partial \pi \chi}+Q_{22} g_{\partial \pi \sigma}+Q_{32} g_{\partial \pi G}, \\
g_{\partial \pi G^{\prime}} & =Q_{13} g_{\partial \pi \chi}+Q_{23} g_{\partial \pi \sigma}+Q_{33} g_{\partial \pi G}, \\
g_{\partial H \pi} & =Q_{11} g_{\partial \sigma \pi}, \\
g_{\partial S \pi} & =Q_{12} g_{\partial \sigma \pi}, \\
g_{\partial G^{\prime} \pi} & =Q_{13} g_{\partial \sigma \pi},
\end{aligned}
$$

and

$$
\begin{aligned}
g_{N G} & =-\frac{b}{\cosh \delta}, \\
g_{\pi G} & =-Z^{2} \frac{\mu^{2}}{G_{0}}+Z^{2} \frac{g_{\chi}}{G_{0}} \chi_{0}, \\
g_{\partial \pi G} & =Z^{2} w^{2}\left(\frac{m_{1}^{2}}{G_{0}}+\frac{g_{\mathrm{AV} \chi_{0}}}{G_{0}}\right) .
\end{aligned}
$$


[1] C. Patrignani et al. (Particle Data Group), Chin. Phys. C 40, 100001 (2016).

[2] S. Godfrey and N. Isgur, Phys. Rev. D 32, 189 (1985).

[3] S. Capstick and N. Isgur, Phys. Rev. D 34, 2809 (1986).

[4] E. van Beveren, T. A. Rijken, K. Metzger, C. Dullemond, G. Rupp, and J. E. Ribeiro, Z. Phys. C 30, 615 (1986).

[5] J. J. Dudek, R. G. Edwards, P. Guo, and C. E. Thomas (for Hadron Spectrum Collaboration), Phys. Rev. D 88, 094505 (2013).

[6] D. J. Wilson, R. A. Briceño, J. J. Dudek, R. G. Edwards, and C. E. Thomas (for the Hadron Spectrum Collaboration), Phys. Rev. D 92, 094502 (2015).

[7] Y. Nambu and G. Jona-Lasinio, Phys. Rev. 122, 345 (1961).

[8] T. Hatsuda and T. Kunihiro, Phys. Rep. 247, 221 (1994).

[9] S. P. Klevansky, Rev. Mod. Phys. 64, 649 (1992).

[10] M.-X. Su, L. Y. Xiao, and H. Q. Zheng, Nucl. Phys. A 792, 288 (2007).

[11] M. K. Volkov, M. Nagy, and V. L. Yudichev, Nuovo Cimento A 112, 225 (1999).

[12] V. Skokov, B. Stokic, B. Friman, and K. Redlich, Phys. Rev. C 82, 015206 (2010).

[13] B.-J. Schaefer, J. M. Pawlowski, and J. Wambach, Phys. Rev. D 76, 074023 (2007).

[14] M. D. Scadron, G. Rupp, and R. Delbourgo, Fortschr. Phys. 61, 994 (2013).

[15] R. Alkofer and L. von Smekal, Phys. Rep. 353, 281 (2001).

[16] G. Eichmann, H. Sanchis-Alepuz, R. Williams, R. Alkofer, and C. S. Fischer, Prog. Part. Nucl. Phys. 91, 1 (2016).

[17] H. Leutwyler, Ann. Phys. (NY) 235, 165 (1994).

[18] J. Gasser and H. Leutwyler, Ann. Phys. (NY) 158, 142 (1984).

[19] S. Scherer, Adv. Nucl. Phys. 27, 277 (2003).

[20] H. W. Fearing and S. Scherer, Phys. Rev. D 53, 315 (1996).

[21] A. V. Manohar, in Perturbative and Nonperturbative Aspects of Quantum Field Theory, Proceedings of the 35. Internationale Universitätswochen für Kern- und Teilchenphysik, Schladming, Austria, March 2-9, 1996, Lecture Notes in Physics Vol. 479 (Springer, Berlin, 1997), p. 311.

[22] G. Ecker, J. Gasser, A. Pich, and E. de Rafael, Nucl. Phys. B 321, 311 (1989).

[23] V. Cirigliano, A. Pich, G. Ecker, and H. Neufeld, J. High Energy Phys. 06 (2003) 012.

[24] P. Ko and S. Rudaz, Phys. Rev. D 50, 6877 (1994).

[25] M. Urban, M. Buballa, and J. Wambach, Nucl. Phys. A 697, 338 (2002).

[26] G. Carter, P. Ellis, and S. Rudaz, Nucl. Phys. A 603, 367 (1996).

[27] D. Parganlija, F. Giacosa, and D. H. Rischke, Phys. Rev. D 82, 054024 (2010).

[28] D. Parganlija, P. Kovács, G. Wolf, F. Giacosa, and D. H. Rischke, Phys. Rev. D 87, 014011 (2013).

[29] P. Kovács, Z. Szép, and G. Wolf, Phys. Rev. D 93, 114014 (2016).

[30] S. Janowski, F. Giacosa, and D. H. Rischke, Phys. Rev. D 90, 114005 (2014)

[31] W. I. Eshraim, S. Janowski, F. Giacosa, and D. H. Rischke, Phys. Rev. D 87, 054036 (2013).

[32] F. Giacosa, J. Sammet, and S. Janowski, Phys. Rev. D 95, 114004 (2017).

[33] D. Parganlija and F. Giacosa, Eur. Phys. J. C 77, 450 (2017).

[34] F. Divotgey, P. Kovacs, F. Giacosa, and D. H. Rischke, Eur. Phys. J. A 54, 5 (2018).
[35] S. Gallas, F. Giacosa, and D. H. Rischke, Phys. Rev. D 82, 014004 (2010).

[36] L. Olbrich, M. Zétényi, F. Giacosa, and D. H. Rischke, Phys. Rev. D 93, 034021 (2016).

[37] C. DeTar and T. Kunihiro, Phys. Rev. D 39, 2805 (1989).

[38] Y. Chen et al., Phys. Rev. D 73, 014516 (2006).

[39] L.-C. Gui, Y. Chen, G. Li, C. Liu, Y.-B. Liu, J.-P. Ma, Y.-B. Yang, and J.-B. Zhang (CLQCD Collaboration), Phys. Rev. Lett. 110, 021601 (2013).

[40] M. Albaladejo and J. A. Oller, Phys. Rev. Lett. 101, 252002 (2008).

[41] F. Brünner, D. Parganlija, and A. Rebhan, Phys. Rev. D 91, 106002 (2015); 93, 109903(E) (2016).

[42] F. Brünner and A. Rebhan, Phys. Rev. Lett. 115, 131601 (2015).

[43] F. Brünner and A. Rebhan, Phys. Rev. D 92, 121902(R) (2015).

[44] C. Amsler and F. E. Close, Phys. Rev. D 53, 295 (1996).

[45] F. E. Close and A. Kirk, Eur. Phys. J. C 21, 531 (2001).

[46] F. Giacosa, T. Gutsche, V. E. Lyubovitskij, and A. Faessler, Phys. Rev. D 72, 094006 (2005).

[47] T. Teshima, I. Kitamura, and N. Morisita, J. Phys. G 30, 663 (2004).

[48] R. J. Jaffe, Phys. Rev. D 15, 267 (1977).

[49] L. Maiani, F. Piccinini, A. D. Polosa, and V. Riquer, Phys. Rev. Lett. 93, 212002 (2004).

[50] G. 't Hooft, G. Isidori, L. Maiani, A. D. Polosa, and V. Riquer, Phys. Lett. B 662, 424 (2008).

[51] A. H. Fariborz, Int. J. Mod. Phys. A 19, 2095 (2004).

[52] M. Napsuciale and S. Rodriguez, Phys. Rev. D 70, 094043 (2004).

[53] O. Krehl and J. Speth, in Hadron Dynamics: New DAФNE and CEBAF Facilities [Nucl. Phys. A 623, 162 (1997)].

[54] L. Li, B.-S. Zou, and G.-1. Li, Phys. Rev. D 63, 074003 (2001).

[55] N. Isgur and J. Speth, Phys. Rev. Lett. 77, 2332 (1996).

[56] M. B. Voloshin and L. B. Okun, Pisma Zh. Eksp. Teor. Fiz. 23, 369 (1976) [JETP Lett. 23, 333 (1976)].

[57] K. Maltman and N. Isgur, Phys. Rev. D 29, 952 (1984).

[58] V. Baru, J. Haidenbauer, C. Hanhart, Yu. Kalashnikova, and A. E. Kudryavtsev, Phys. Lett. B 586, 53 (2004).

[59] T. Branz, T. Gutsche, and V. E. Lyubovitskij, Eur. Phys. J. A 37, 303 (2008).

[60] J. R. Pelaez, Phys. Rev. Lett. 92, 102001 (2004).

[61] J. A. Oller and E. Oset, Nucl. Phys. A 620, 438 (1997); 652, 407 (1999).

[62] J. R. Pelaez, Phys. Rep. 658, 1 (2016).

[63] M. Boglione and M. R. Pennington, Phys. Rev. D 65, 114010 (2002).

[64] D. Morgan and M. R. Pennington, Phys. Rev. D 48, 1185 (1993).

[65] N. A. Tornqvist, Z. Phys. C 68, 647 (1995).

[66] N. A. Tornqvist and M. Roos, Phys. Rev. Lett. 76, 1575 (1996).

[67] E. van Beveren, D. V. Bugg, F. Kleefeld, and G. Rupp, Phys. Lett. B 641, 265 (2006).

[68] T. Wolkanowski, F. Giacosa, and D. H. Rischke, Phys. Rev. D 93, 014002 (2016).

[69] T. Wolkanowski, M. Sołtysiak, and F. Giacosa, Nucl. Phys. B 909, 418 (2016).

[70] S. Gallas, F. Giacosa, and G. Pagliara, Nucl. Phys. A 872, 13 (2011). 
[71] A. Heinz, S. Struber, F. Giacosa, and D. H. Rischke, Phys. Rev. D 79, 037502 (2009).

[72] W. Deinet, K. Teilab, F. Giacosa, and D. H. Rischke, Phys. Rev. C 94, 044001 (2016).

[73] D. Jido, M. Oka, and A. Hosaka, Prog. Theor. Phys. 106, 873 (2001).

[74] D. Jido, Y. Nemoto, M. Oka, and A. Hosaka, Nucl. Phys. A 671, 471 (2000).

[75] D. Zschiesche, L. Tolos, J. Schaffner-Bielich, and R. D. Pisarski, Phys. Rev. C 75, 055202 (2007).

[76] S. Gallas and F. Giacosa, Int. J. Mod. Phys. A 29, 1450098 (2014).

[77] C. Sasaki and I. Mishustin, Phys. Rev. C 82, 035204 (2010).

[78] S. Benic, I. Mishustin, and C. Sasaki, Phys. Rev. D 91, 125034 (2015).

[79] W.-G. Paeng, H. K. Lee, M. Rho, and C. Sasaki, Phys. Rev. D 85, 054022 (2012).

[80] F. Giacosa, A. Koenigstein, and R. D. Pisarski, Phys. Rev. D 97, 091901(R) (2018).

[81] A. Salomone, J. Schechter, and T. Tudron, Phys. Rev. D 23, 1143 (1981).

[82] A. A. Migdal and M. A. Shifman, Phys. Lett. B 114, 445 (1982).

[83] H. Gomm and J. Schechter, Phys. Lett. B 158, 449 (1985).

[84] H. Gomm, P. Jain, R. Johnson, and J. Schechter, Phys. Rev. D 33, 801 (1986).

[85] F. Giacosa, Phys. Rev. D 75, 054007 (2007).

[86] R. D. Pisarski and V. V. Skokov, Phys. Rev. D 94, 054008 (2016).

[87] A. H. Fariborz, R. Jora, and J. Schechter, Phys. Rev. D 72, 034001 (2005).
[88] A. H. Fariborz, R. Jora, J. Schechter, and M. N. Shahid, Phys. Rev. D 83, 034018 (2011).

[89] A. De Rujula, H. Georgi, and S. L. Glashow, Phys. Rev. D 12, 147 (1975).

[90] G. 't Hooft, Phys. Rev. D 14, 3432 (1976).

[91] E. V. Shuryak, Nucl. Phys. B 203, 93 (1982).

[92] U. Vogl and W. Weise, Prog. Part. Nucl. Phys. 27, 195 (1991).

[93] P. Maris and C. D. Roberts, Int. J. Mod. Phys. E12, 297 (2003).

[94] D. Bugg, Eur. Phys. J. C 52, 55 (2007).

[95] B. Peyaud, in Proceedings of the 8th International Conference on Beauty, Charm and Hyperons in Hadronic Interactions [Nucl. Phys. B 187, 29 (2009)].

[96] V. Baru, C. Hanhart, M. Hoferichter, B. Kubis, A. Nogga, and D. Phillips, Phys. Lett. B 694, 473 (2011).

[97] V. Baru, C. Hanhart, M. Hoferichter, B. Kubis, A. Nogga, and D. Phillips, Nucl. Phys. A 872, 69 (2011).

[98] H.-C. Schröder, A. Badertscher, P. Goudsmit, M. Janousch, H. Leisi, E. Matsinos, D. Sigg, Z. Zhao, D. Chatellard, J.-P. Egger, K. Gabathuler, P. Hauser, L. Simons, and A. Rusi El Hassani, Eur. Phys. J. C 21, 473 (2001).

[99] D. Gotta et al., in Precision Physics of Simple Atoms and Molecules, Lecture Notes in Physics Vol. 745 (Springer, Berlin, 2008).

[100] T. Matsui and B. Serot, Ann. Phys. (NY) 144, 107 (1982).

[101] L. Schneider, Bachelors' thesis, Institute for theoretical physics Goethe University Frankfurt, 2018 (unpublished).

[102] https://github.com/Phillip2/eLSM

[103] L. Olbrich, M. Zétényi, F. Giacosa, and D. H. Rischke, Phys. Rev. D 97, 014007 (2018).

[104] B. Ananthanarayan, G. Colangelo, J. Gasser, and H. Leutwyler, Phys. Rep. 353, 207 (2001).

[105] E. T. Osypowski, Nucl. Phys. B 21, 615 (1970). 